\title{
FACTORS AFFECTING ULTRAVIOLET SOLAR-RADIATION INTENSITIES
}

\author{
By W. W. Coblentz and R. Stair
}

\section{ABSTRACT}

Ultraviolet-filter transmission measurements are made, using a newly devised portable precision ultraviolet meter (Research Paper RP647) and 4 glass filters, giving the special quality and total intensity in the band of ultraviolet solar radiation extending from about 2900 to $3500 \mathrm{~A}$. At the high-altitude station (Flagstaff, Ariz.) data were obtained on solar ultraviolet intensities under various conditions; high and low humidity, large and small air masses, clear and smoky skies. The data obtained at sea-level stations (Washington, D. C., and San Juan, P. R.) are of interest in connection with the question of ultraviolet intensities in the tropies as compared with similar stations at higher latitudes.

Data are given on the amount of ultraviolet transmitted through light fog, and on the amount reflected by the sky and by snow; also the effect of altitude and latitude on the solar ultraviolet intensity at the earth's surface.

The observations show that, while a light fog greatly reduces the intensity (when the sun appeared as a distinctly outlined white disk, the intensity was reduced to one-tenth or less) the spectral quality was practically unchanged, owing to the relatively nonselective character of the transmission through water vapor. Similarly, the ultraviolet reflected from snow is extraordinarily high, owing to the fact that the ice crystals, within which the reflection occurs, are highly transparent to ultraviolet radiation.

The absorption of ultraviolet is caused by atmospheric polution near the earth's surface, and by ozone in the stratosphere. An increase of 40 to 50 percent in intensity in the band of wave length between 2900 and $3130 \mathrm{~A}$, is observed in rising $2 \mathrm{~km}(7,000 \mathrm{ft})$ above sea level. A much greater increase in intensity may be expected at elevations of 15 to $25 \mathrm{~km}$ (10 to 15 miles). How. ever, such heights are not practicable for large-scale biological tests.

The measurements at the Flagstaff station show a greater atmospheric transparency (less ozone) in the afternoon than in the forenoon; and a greater transparency in the autumn than in the spring. This is in agreement with the work of Dobson and others, using other methods of observation.

For the same solar altitude (air mass traversed by the rays) the ultraviolet solar intensities in the tropies (at San Juan) were somewhat higher than at a midlatitude, sea-level station (Washington) that is free from local air pollution. This is in agreement with other observations showing that, in the tropics, for the same solar altitude, the amount of atmospheric ozone is less, and consequently the ultraviolet intensities should be somewhat higher than in higher latitudes.

However, this small difference in intensity, for the same solar altitude, does not appear to be sufficient to produce marked differences in biological effects. If differences in biological effects ascribable exclusively to a difference in ultraviolet intensities are observable, they may be owing to the fact that for about 4 months (April 20 to August 20) at the noon hour, in the tropics, the sun shines through an air mass of $m=1.00$ to 1.01 , as compared with an air mass of $m=1.04$ to 1.05 in midlatitude (Washington). Consequently, during this period, the shortest-biologically most effective-wave lengths (at 2900 to $3000 \mathrm{~A}$ ) are very greatly increased in intensity. The average intensity of the whole spectral band (at 2900 to $3130 \mathrm{~A}$ ), recognized as having a specific biologic action, at least in curing rickets) during the time when the sun shines through an air mass of $m=1.00$ to 1.01 , is about 20 percent higher in the tropics than is ever attained in midlatitude. 
Similarly, during the 5 winter months, at midlatitude stations, the shortestbiologically most effective-ultraviolet wave lengths are almost completely absorbed, and the intensity of the less effective wave lengths is less than one-sixth the value that obtains in the tropies.

In addition to the greater intensity of the ultraviolet, there, usually is also a greater number of hours of sunshine in the tropics during the winter months. Other factors to be considered are differences in air movement, temperature, humidity, and the amount of clothing worn, all of which factors have an effect upon the incidence of rickets, which is practically unknown in the tropics.

\section{CONTENTS}

I. Purpose and simplified method of ultraviolet solar radiometry _....-

II. Climatic conditions at the observing stations _._.

III. Minor observations _._.

1. Fog _.

2. Snow

3. Sky _......... 142

4. Erythema tests

IV. Effect of altitude on ultraviolet solar radiation $\ldots \ldots$

V. Effect of geographic latitude on ultraviolet solar radiation _...... 147

VI. References_... 150

\section{PURPOSE AND SIMPLIFIED METHOD OF ULTRAVIOLET SOLAR RADIOMETRY}

A knowledge of the intensity and spectral quality of short wave length ultraviolet solar radiation is of great importance in climatology, physiology, and biology.

The interest of the National Bureau of Standards in this subject is in methods of exact evaluation of the spectral band of ultraviolet solar radiation, of wave lengths extending from about $3200 \mathrm{~A}$ to the limit of transparency of the earth's atmosphere at about $2900 \mathrm{~A}$, which is recognized as having a specific effect in preventing and healing rickets, and on this basis is being used as an adjunct in medicine.

The object of the present communication is to describe measurements of the spectral quality of short wave length ultraviolet solar radiation, at different stations, as affected by the altitude of the sun, by the geographic latitude and the altitude of the observing station, and by different atmospheric conditions. In a future communication a description will be given of recent developments in the exact evaluation of ultraviolet radiation in absolute units.

The ultraviolet intensities are measured by means of a newly developed radiometer (1) ${ }^{1}$ consisting of a titanium photoelectric cell, which responds only to the spectral band under consideration, and a balanced amplifier (fig. 1) for measuring the weak photoelectric currents induced in the photoelectric cell by ultraviolet radiation. By means of a group of four glass filters this spectral band of ultraviolet (extending from about $2900 \mathrm{~A}$ to $3500 \mathrm{~A}$ ) is separated into 5 parts. In this manner it is possible to determine, in a few minutes, the shape of this part of the solar spectral-energy curve, apparently as accurately as has thus far been possible with a spectroradiometer. The latter method consumes a much longer time to make the observations, which, in addition, require numerous corrections, including adjustments for changes in atmospheric transmission during the measurements.

1 The figures in parentheses here and elsewhere in the text refer to the literature references at the end of this paper. 


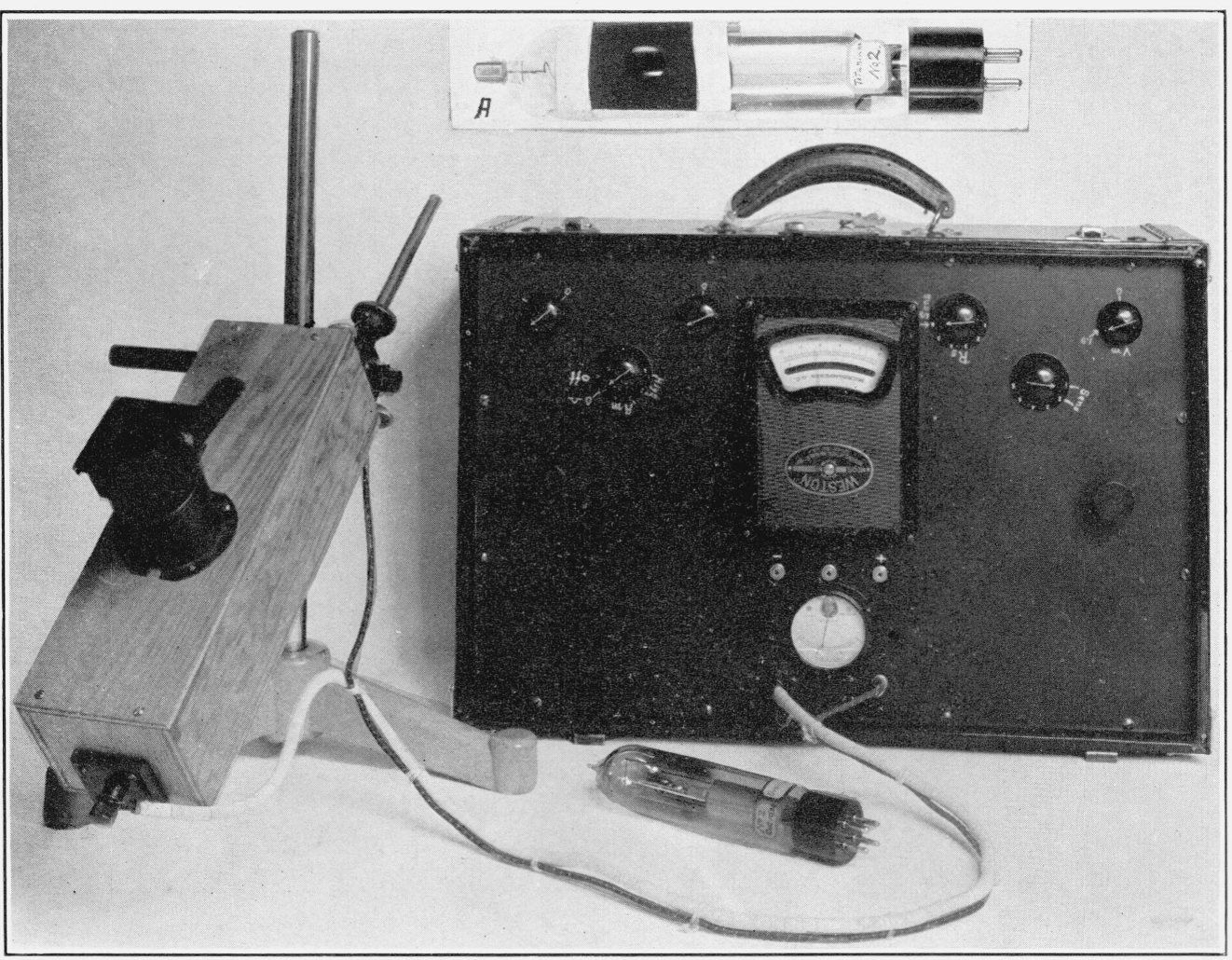

Figure 1.-Ultraviolet meter.

This consists of a titanium photoelectric cell (left, mounted on polar axis; unmounted cell in center foreground and in inset $A$ ) and balanced amplifier. 


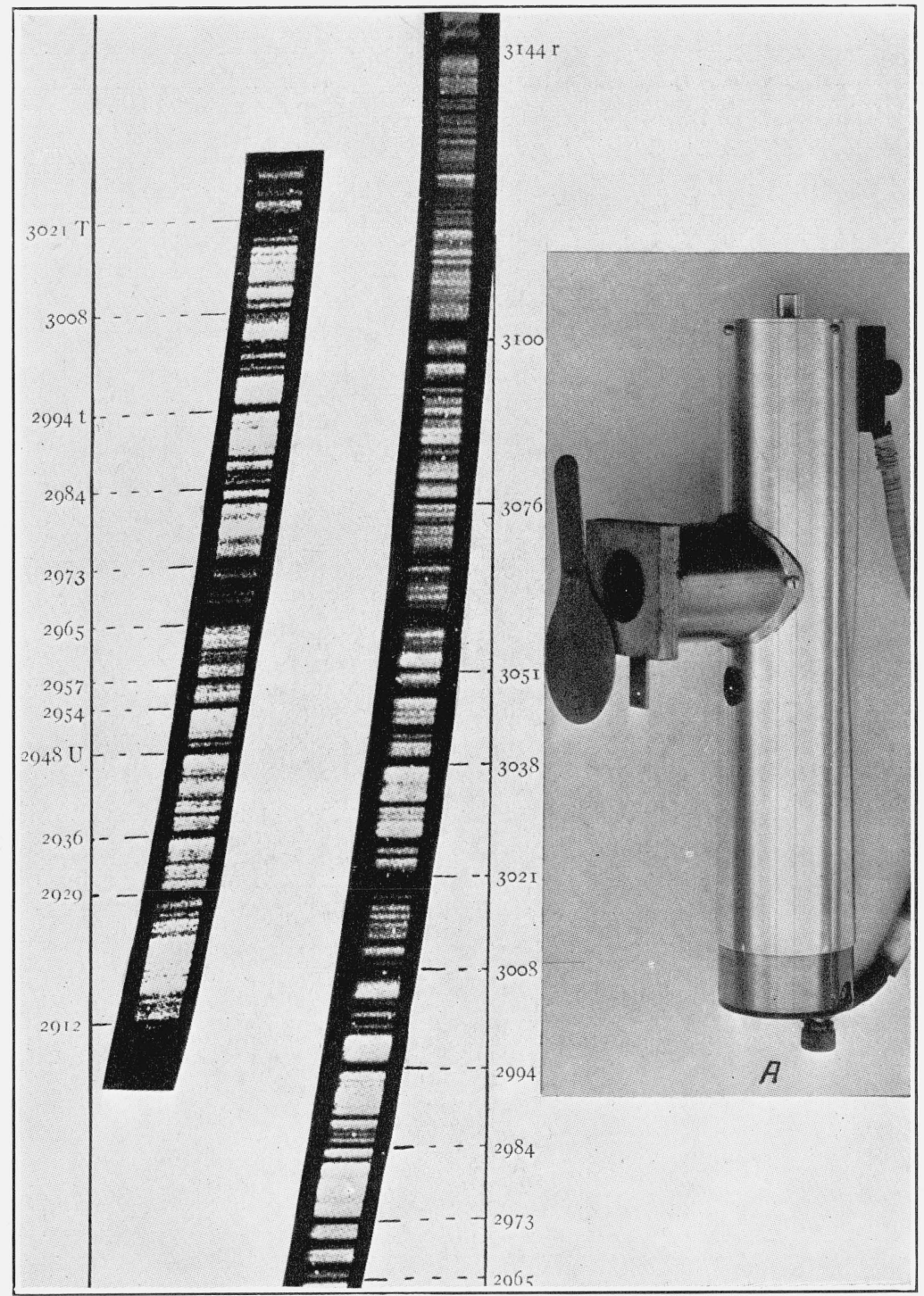

FigURE 2.-Ultraviolet end of the solar spectrum, obtained by Fabry and Buisson, at Marseille in June 1920.

By means of a focal-plane shutter, which occulted different parts of the spectrum, in going from 3020 to $2930 \mathrm{~A}$ the time of the exposure was increased in the ratio of from 1 to $1,000$. $A$ is a metal container for the photoelectric cell (see fig. 1). 
In both methods, wide bands of the spectrum are measured. Hence, owing to the small dispersion that can be used, the observed spectral-energy curve, in the region of 2900 to $3500 \mathrm{~A}$ appears to be relatively smooth and free from indentations (2); whereas, using a high dispersion and a narrow slit, photographs (3) show the spectrum deeply indented with absorption lines, caused by vapors of various elements in the solar atmosphere and by varying degrees of concentration of ozone in the terrestrial atmosphere (fig. 2). Recalling (3) the fact that the intensity of solar radiation at $3143 \mathrm{~A}$ is about 1 million times that at $2898 \mathrm{~A}$, and that the intensity throughout this whole spectral region is extremely low, it will be evident to the reader that it is practically impossible to obtain, radiometrically, a solar spectral-energy curve, accurately showing the indentations by the absorption lines indicated in the spectrum photographs.

Hence, for the purpose of the present work, the evaluation of the spectral-energy curve that gives calculated filter transmissions which are in agreement with the observed values is probably as accurate as the spectral-energy curve determined directly with a spectroradiometer. In fact, from the highly selective response of the photoelectric cell in the region of 2900 to $3000 \mathrm{~A}$, slight variations in ultraviolet intensity in this spectral region (in which we are especially interested) are more easily and more accurately determined by the integrated measurements with the filters than with a spectroradiometer, because of the accompanying losses in intensity by absorption in the optical system and in the auxiliary reflecting apparatus.

The experimental procedure of the investigation now in progress consists in observing the variation in the total intensity of the ultraviolet radiation in the band of wave lengths extending from about 2900 to about $3500 \mathrm{~A}$ in sunlight, throughout the day and throughout the year, by means of titanium photoelectric cells having the spectral response curves illustrated in figure 3. At the same time, the percentage transmission of this band of radiation through a set of four glass filters (designated Corex D, Nillite, Ba-flint-1, and Baflint-3, having the spectral-transmission curves illustrated in fig. 3) is determined. The variation in transmission of this band of ultraviolet radiation, through any one filter, with variation in solar altitude, air pollution, geographic latitude, etc., indicates a variation in spectral quality - the higher the transmission, the lower the intensity of the radiation of the shortest wave lengths.

Data of this type are illustrated in figures 4 and 5, which depict the observations made at the Lowell Observatory, Flagstaff, Ariz. (elevation 7,300 ft.) during the summer of 1934 .

The next step is to determine, by calculation, the shape of the spectral-energy curve required to give the observed transmissions of ultraviolet through the 4 filters. For this purpose, in the first trial calculation, use was made of the average ultraviolet solar spectral energy curve observed by Pettit (2) at Tucson, Ariz. (elevation about $2,500 \mathrm{ft}$ ), in May 1931. The product of this spectral-energy curve and the spectral response curve of the photoelectric cell (fig. 3) gives the solar spectral-energy curve as it affects the particular photoelectric cell employed in making the measurements (fig. 6).

For example, curve "Ti No. 1" in figure 6 is the shape of the solar spectral-energy curve as it would be observed by the uncovered titanium photoelectric cell no. 1. The product, at each wave length, 


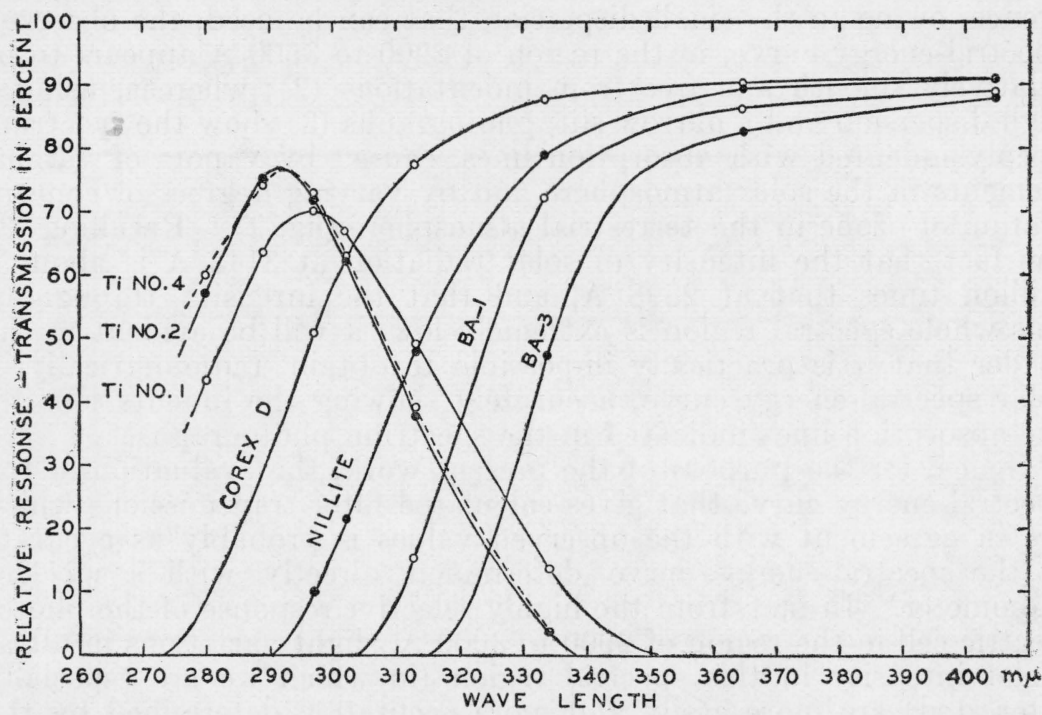

FIG. 3.-Relative response curves of titanium photoelectric cells nos. 1, 2, and 4, to 1. an equal-energy spectrum; also spectral transmission of the glass filters usedCorex D, Nillite (both trade names), and barium-flint glass ( $\mathrm{Ba}-1$ and $\mathrm{Ba}-3$, thickness $1 \mathrm{~mm}$ and $3 \mathrm{~mm}$, respectively).

Wave lengths $400 \mathrm{~m} \mu=4000 \mathrm{~A}$

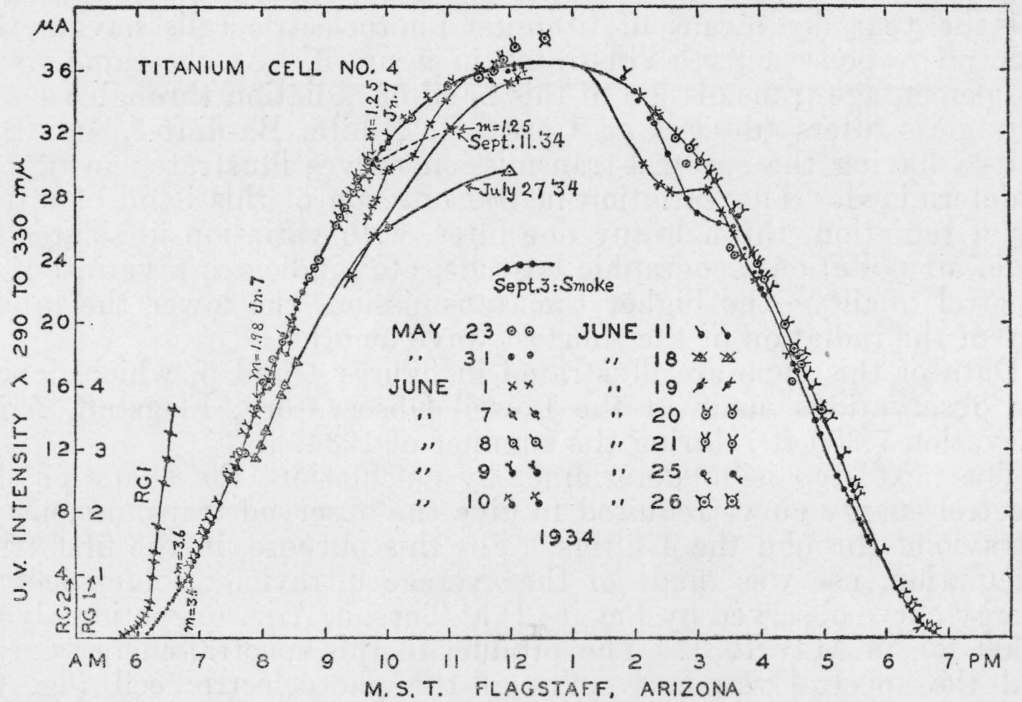

FIG. 4.-Variation in ultraviolet solar intensities throughout the day (MST= mountain standard time) and as affected by smoke.

The ordinates are readings on the micrometer; amplifier sensitivity, Rg. $1=1.57 \mathrm{Rg}$. 2. Observations made at Flagstaff, Ariz., elevation $7,300 \mathrm{ft}$. 
of this spectral energy by the corresponding percentage transmission of the filter (fig. 3 ) gives the spectral-energy curves marked Cx., Ni., $\mathrm{Ba}-1$, and $\mathrm{Ba}-3$, in figure 6 . The ratios of the areas under these curves to the area under the curve, Ti No. 1, gives the calculated

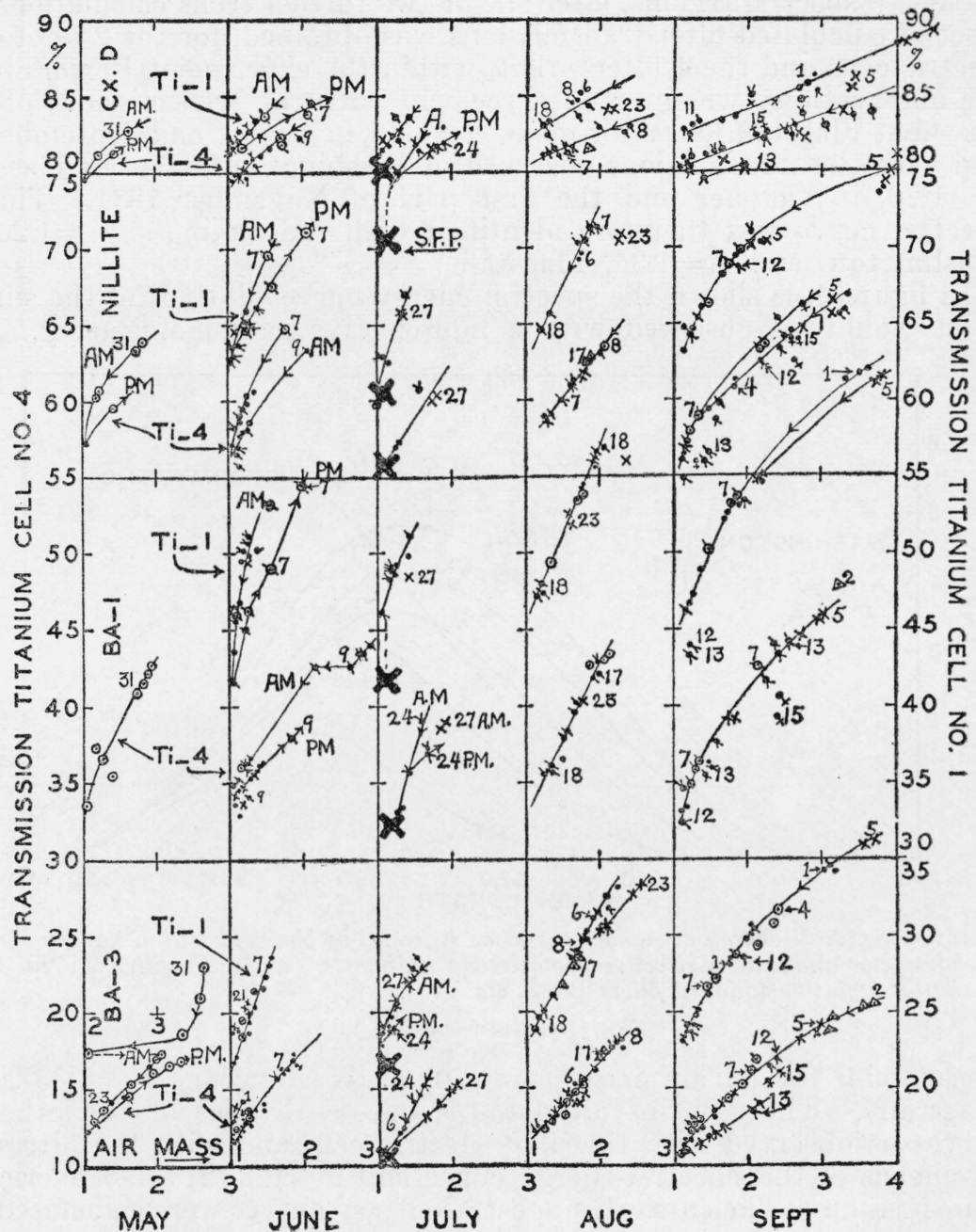

FIG. 5.-Variation in transmission of ultraviolet solar radiation through the various filters (Ba-3, etc.) with solar altitude (air mass, $m=1,2$, 3), time of the day (a.m.; p.m.) and the season, as observed with 2 photoelectric cells (Ti-1 upper sets of curves; Ti-4, lower sets of curves (see fig. 3 for spectral response)).

The numbers $(1,7,24$, etc.) are the dates of observation. The heavy crosses ( $x \times x$, S.F.P.) indicate observations on the San Francisco peaks (Fremont Saddle, elevation 10,500 ft).

percentage of ultraviolet transmitted through the filters. Similar curves were obtained for the titanium photoelectric cell no. 4 .

From the deviations of the calculated from the observed filter transmissions, which were in agreement for both photoelectric cells, it was apparent that the spectral intensities indicated in Pettit's 
energy curve, in the region of 2950 to $3050 \mathrm{~A}$, were slightly too high, and the intensities at $3250 \mathrm{~A}$ to $3550 \mathrm{~A}$ were appreciably too low, to fit the observations of 1934 at the higher elevation $(7,300 \mathrm{ft}$.) at Flagstaff, Ariz. By making slight changes in the energy curve in these two spectral regions, after one or two further trials calculations, a set of calculated filter transmissions was obtained, for the 2 photoelectric cells and the 4 filters, that, within the experimental errors in the observations, was in good agreement with the transmissions observed at Flagstaff for an air mass, $m=1.35$, in August and September and with the transmissions observed in Washington for an air mass, $m=1.20$, in October and the first part of November 1934. This spectral curve was therefore identified with the air mass, $m=1.20$, Washington, and $m=1.35$, Flagstaff.

In figure 6 , is shown the spectral energy curve (dotted) of the sun as it would be observed with a nonselective radiometer (e. g., a

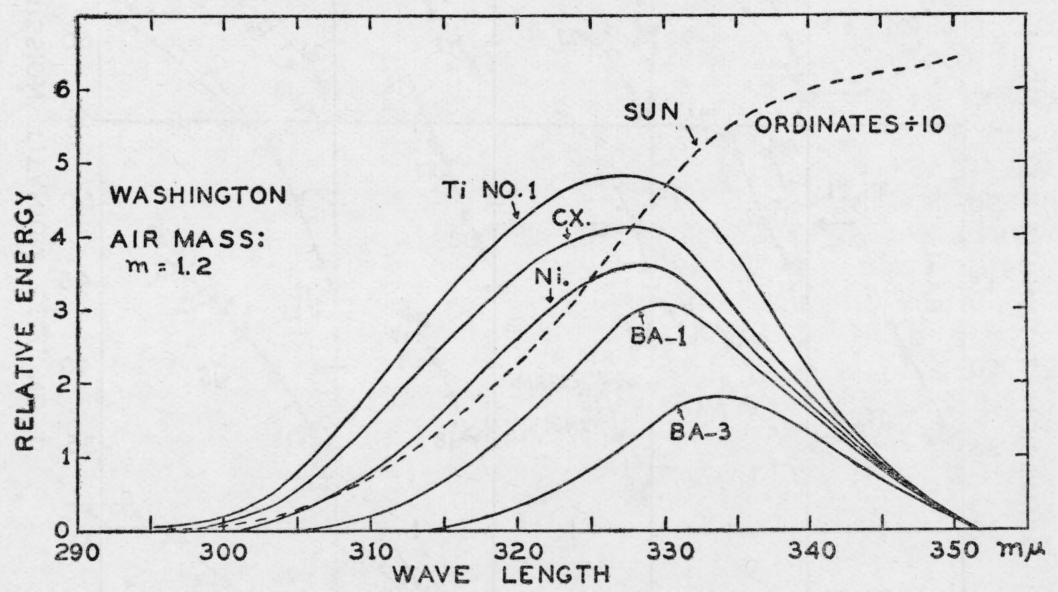

Fig. 6.-Spectral-energy curve of the sun as it would be observed with a nonselective radiometer and with a selective photoelectric radiometer, without a filter, Ti No. 1, and through the standard filters $\mathrm{Ba}-3$, etc.

\section{Wave lengths $350 \mathrm{~m} \mu=3500 \mathrm{~A}$}

thermopile) for an air mass, $m=1.20$, at Washington $(m=1.35$ at Flagstaff). The scale of ordinates is one-tenth that of the other curves as observed with the photoelectric cell, and with the filters. By means of this spectral-energy curve and Pettit's (2) atmospherictransmission coefficients other spectral-energy curves were calculated. On multiplying, point by point, these spectral-energy curves by the spectral-response curves of the 2 photoelectric cells and by the spectral transmissions of the respective filters, as indicated above (see fig. 3) calculated transmissions were obtained for the 4 filters, using 2 photoelectric cells, which (after one or two trial adjustments of the assumed spectral-energy curve) were in close agreement with the observed filter transmissions for air masses $m=1.05,1.10,1.25$, 2.20 , and 3.05 at Flagstaff, and with the observed transmissions for correspondingly smaller air masses in Washington.

In view of the fact that the atmospheric transparency, for a given air mass, may vary from morning to afternoon, with the season, and with the ultraviolet emission of the sun (as affected by sun spots), no attempt is made to identify the calculated with the observed filter 
transmissions closer than air mass $\Delta m=0.05$. If great refinements are attempted, the spectral-energy curve corresponding with the filter transmissions, observed at the time of the physiological tests, should be used instead of a standard curve corresponding with the solar altitude at the time of the test.

The ultraviolet spectral-energy curve, calculated in this manner to fit the observed filter transmission for a certain air mass, appears to be sufficiently accurate for use in the evaluations desired. If the method should be adopted for general use, only 1 or 2 filters, having integral transmissions of 30 to 60 percent, would be necessary; although it is instructive to use an additional filter that transmits 80 percent. For example, in a recent test (January 2, 1935) in Washington, although the air was very clear, ${ }^{2}$ the transmission of the Corex-D filter remained practically constant throughout the day, showing that, even in the clearest weather, the amount of ultraviolet of wave lengths shorter than $3020 \mathrm{~A}$ in midlatitude, sea-level, winter sunlight is extremely small.

It is to be noted that this method gives the relative (intensity) energy distribution for a given air mass, which information is sufficient for evaluating the integrated energy in a particular spectral band (6), (16), (17). The ordinates (intensities) of the spectralenergy curves for different air masses are on an arbitrary scale, which need not be the same for all the curves. By making supplementary observations and calculations, these curves can be placed on an absolute energy basis.

It is to be noted also that much information on the transparency of the atmosphere can be obtained from a comparison of the amount of ultraviolet transmitted through the filters. For a given air mass (say $m=1.1$ ) the lowest filter transmissions (the highest short wave length ultraviolet intensities), observed through the clearest skies, indicate the least amount of ozone. Conversely the highest filter transmissions, observed through a clear sky (e. g., as observed on January 24,1935 ), are ascribable to a high ozone content. Usually when the atmosphere is polluted with smoke and dust, the filter transmissions are high. In this case, no attempt is made to determine how much of this high transmission is owing to the presence of smoke and dust in the lower atmosphere and how much is owing to ozone-both sources having selective absorption in the short wave length ultraviolet.

On September 3, 1934, the filter transmissions observed through a smoky atmosphere, contrary to expectations, were the same as for clear weather at that season. The interpretation to be given is that this smoke was nonselective in transmission and that the ozone content was low (the ultraviolet intensity in the shortest wave lengths was high) in accordance with the season.

At the higher altitude (Flagstaff) local, almost imperceptible, changes in atmospheric transparency are easily recorded by ultraviolet radiometry. For example, on September 13, 1934, which was one of the clearest days of the season, the ultraviolet filter transmissions were very low, corresponding with a low ozone content (a high

${ }^{2}$ One criterion for judging clearness is a low-hanging, dark haze on the horizon, NNE. of the National Bureau of Standards, visible on the clearest days, and apparently identified with the "city haze" over Baltimore, some 35 miles away. 
intensity of short wave length ultraviolet). The sky radiation was low. Nevertheless, owing to an imperceptible temporary, local pollution (or more likely an increase in ozone at the noon hour) the filter transmissions were perceptibly higher at the noon hour than at 10 a.m.

It is to be noted that a slight difference in the spectral response curves of two photoelectric cells has a noticeable effect upon the filter transmissions when the measurements are made under constant atmospheric conditions. For example, as illustrated in figure 3 , the photoelectric cells Ti No. 2 and Ti No. 4, differ but little in spectral response-Ti No. 4 apparently being slightly more sensitive in the shortest wave lengths and slightly less sensitive in the longer wave lengths. The filter transmissions observed with photoelectric cell Ti No. 4 should, therefore, be slightly lower than with Ti No. 2. Careful measurements on the filter, $\mathrm{Ba}-3$, through practically the same air mass (September 11, 1934, $m=1.22$ ) gave a transmission of 12.2 percent using Ti No. 4 , and 13.1 percent using Ti No. 2, in agreement with expectations.

It is impracticable to discuss all the observed data, some of which are given in table 1. A study of these data reveals interesting information indicating the constancy of atmospheric conditions as shown by the same percentage transmissions of the filters for the same solar altitudes, on widely different dates; for example (table 1, colums 7 and 8) the measurements on June 22 and July $6, m=1.27$; also the early morning measurements on June 9 and August $8,1934, m=2.04$. Again, at 8 to 9 a.m., the sky was clearer on September 12 than on September 7, giving the same filter transmissions for larger air masses.

At high altitudes slight changes in atmospheric conditions produce marked changes in the filter transmissions, as noted on September 13 (one of the clearest days) as compared with September 12 and 14, 1934. August 27 and September 5 are typical of sudden changes in sky conditions. June 10 was typical of a low humidity (press aq. vap. $=1.58 \mathrm{~mm}$ ) and the attendant difficulty of keeping the filters free from fine dust which was attracted by electric charges on the glass. June 9 was a good day to obtain data from early morning to late afternoon (fig. 4). 
TABLE 1.-Filter transmissions of integrated ultraviolet solar radiation for different air masses, $m$, using a titanium photoelectric cell, Ti No. 4 (see fig. 3).

[Filters: Ba-3=barium flint, thickness $3.12 \mathrm{~mm} ; \mathrm{Ba}-1$, same, thickness $1.00 \mathrm{~mm} ; \mathrm{Ni} .=$ Nillite (trade name); $\mathrm{Cx} .=\mathrm{Corex} \mathrm{D}$ (trade name) (see fig. 3 ). Tr $=$ percentage transmission. The intensity is the microammeter-scale reading ( $\mu a)$ for Rg. 2 , and amplifier sensitivity $=12.1$. Observations made at Flagstaff, Ariz. (latitude $35^{\circ} 12^{\prime} \mathrm{N}$, altitude $7,300 \mathrm{ft}$ ), in 1934 The time is mountain standard time; 26 min, 45 sec faster than local time. The dagger, $t$, and asterisk, *, are used to associate intensity with air mass]

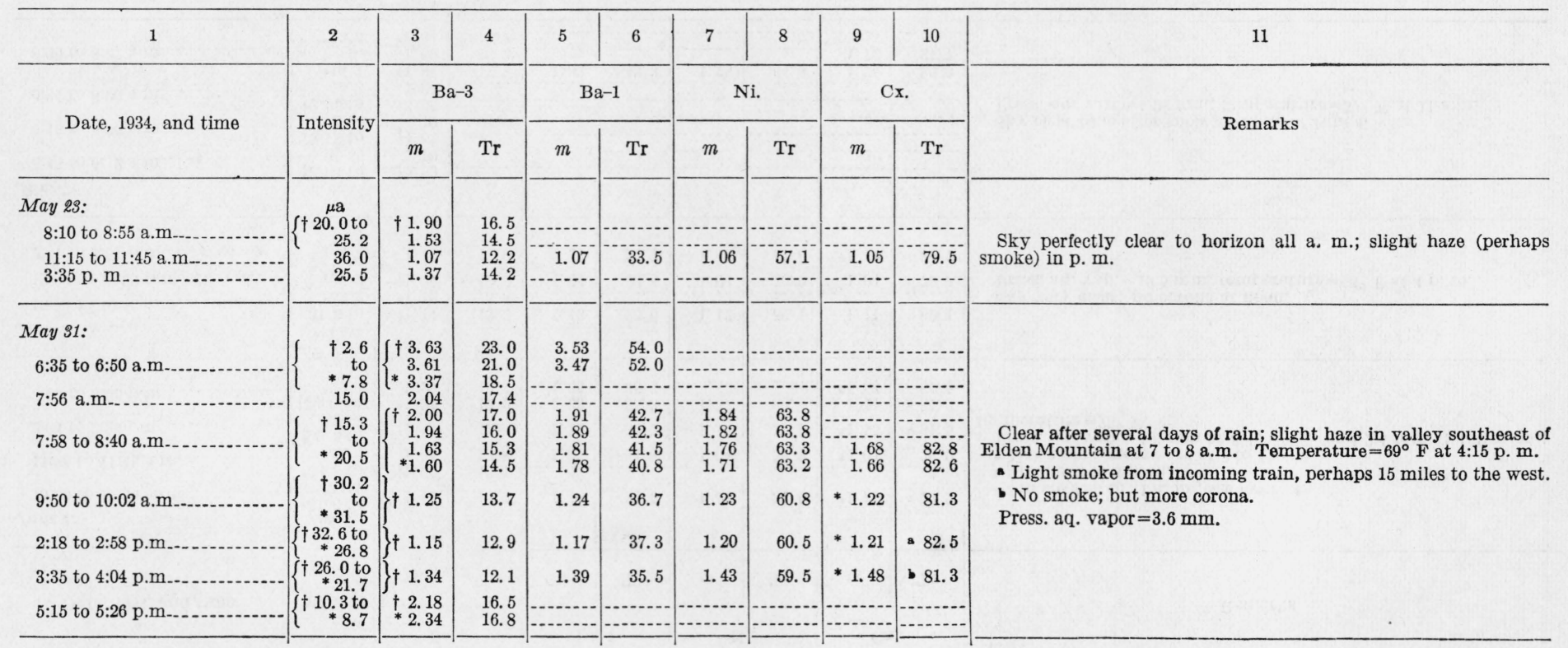


TABLE 1.-Filter transmissions of integrated ultraviolet solar radiation for different air masses, m, using a titanium photoelectric cell, Ti No. 4 (see fig. 3)-Continued

\begin{tabular}{|c|c|c|c|c|c|c|c|c|c|c|}
\hline 1 & 2 & 3 & 4 & 5 & 6 & 7 & 8 & 9 & 10 & 11 \\
\hline \multirow{2}{*}{ Date, 1934 , and time } & \multirow{2}{*}{ Intensity } & \multicolumn{2}{|c|}{$\mathrm{Ba}-3$} & \multicolumn{2}{|c|}{$\mathrm{Ba}-1$} & \multicolumn{2}{|c|}{ Ni. } & \multicolumn{2}{|c|}{ Cx. } & \multirow{2}{*}{ Remarks } \\
\hline & & $m$ & $\mathrm{Tr}$ & $m$ & $\operatorname{Tr}$ & $m$ & $\operatorname{Tr}$ & $m$ & $\operatorname{Tr}$ & \\
\hline $\begin{array}{l}\text { June } 1: \\
\text { 10:29 to } 10: 46 \text { a.m.-. } \\
\text { 11:25 to } 11: 48 \text { a.m.- } \\
\text { 2:04 to } 2: 34 \text { p.m. } \\
\text { 3:09 to } 3: 35 \text { p.m. }\end{array}$ & 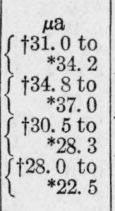 & $\left\{\begin{array}{r}\dagger \\
\dagger 1.15 \\
\dagger 1.06 \\
1.04 \\
\dagger 1.11 \\
\dagger 1.25 \\
1.29\end{array}\right.$ & \begin{tabular}{l|}
14.3 \\
13.1 \\
12.8 \\
12.4 \\
12.2 \\
12.5
\end{tabular} & $\begin{array}{l}1.05 \\
1.12 \\
1.33 \\
1.35\end{array}$ & $\begin{array}{l}34.8 \\
32.7 \\
35.3 \\
35.7\end{array}$ & $\begin{array}{r}1.12 \\
\hdashline 1.04 \\
1.14 \\
1.37 \\
\hdashline\end{array}$ & $\begin{array}{r}58.6 \\
56.8 \\
57.8 \\
60.6 \\
\hdashline-.-\end{array}$ & $\begin{array}{l}* 1.11 \\
* *^{*} 1.04 \\
* 1.15 \\
1.41 \\
*_{1.53}\end{array}$ & $\begin{array}{r}\text { a } 79.2 \\
78.9 \\
\text { b } 81.5 \\
\text { - } 81.0 \\
81.5\end{array}$ & $\begin{array}{l}\text { a Smoke } 9: 30 \text { to } 11 \text { a. m., see figure } 4 \text {. } \\
\text { S Smoke, } 2 \text { to } 3 \text { p. m. } \\
\text { o No smoke; more haze at } 3 \text { to } 4 \text { p. m. } \\
\text { Press. aq. vap. }=4.6 \mathrm{~mm} \text { at } 8: 45 \mathrm{a} \text {. m., and } 2.4 \mathrm{~mm} \text { at } 4: 25 \mathrm{p} \text {. m.; } \\
\text { temperature }=73^{\circ} \mathrm{F} \text {. }\end{array}$ \\
\hline $\begin{array}{l}\text { June } 3: \\
\text { 10:30 to } 10: 53 \text { a.m. } \\
\text { 12:04 to } 12: 20 \text { p.m. } \\
2: 31 \text { to } 2: 51 \text { p.m. }\end{array}$ & $\left\{\begin{array}{r}\uparrow 31.3 \text { to } \\
* 32.2 \\
\dagger 3.2 \\
29.8 \text { to } \\
* 23.7\end{array}\right.$ & $\begin{array}{r}\dagger 1.14 \\
+1.10 \\
\dagger 1.04 \\
1.16\end{array}$ & $\begin{array}{l}13.3 \\
13.8 \\
12.0 \\
13.0\end{array}$ & $\begin{array}{l}1.04 \\
1.17\end{array}$ & \begin{tabular}{l|}
36.0 \\
30.6 \\
29.7
\end{tabular} & $\begin{array}{l}1.12 \\
1.04 \\
1.18\end{array}$ & $\begin{array}{l}56.9 \\
57.8\end{array}$ & $\begin{array}{r}1.11 \\
{ }^{*} 1.04 \\
1.19\end{array}$ & $\begin{array}{l}82.1 \\
79.5 \\
80.7\end{array}$ & $\begin{array}{l}\text { Sky very clear; no corona at noon. } \\
\text { Press. aq. vap. }=19.5 \mathrm{~mm} \text {; temperature }=66^{\circ} \mathrm{F} \text { at } 4 \mathrm{p} . \mathrm{m} \text {. }\end{array}$ \\
\hline $\begin{array}{l}\text { June } 7: \\
\text { 5:45 to } 6: 12 \text { a.m.- } \\
6: 15 \text { to } 6: 30 \text { a.m.- } \\
6: 34 \text { to } 8: 00 \text { a.m... } \\
\text { 8:05 to } 8: 32 \text { a.m. }\end{array}$ & $\left\{\begin{array}{r}\dagger 0.01 \text { to } \\
0.35 \\
\dagger 0.78 \text { to } \\
2.02 \\
\dagger 2.35 \text { to } \\
14.8 \\
\dagger 16.1 \\
\text { to } \\
* 19.6\end{array}\right.$ & $\begin{array}{r}\dagger 5.80 \\
4.70 \\
\dagger 4.65 \\
4.10 \\
\dagger 3.95 \\
1.95 \\
\dagger 1.88 \\
1.69 \\
* 1.64\end{array}$ & $\begin{array}{l}17.3 \\
16.3 \\
15.7\end{array}$ & 1.83 & 43.3 & 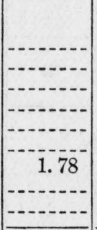 & 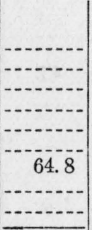 & $\begin{array}{r}1.75 \\
1.72 \\
-1.7\end{array}$ & $\begin{array}{r} \\
\\
\\
\\
\end{array}$ & $\begin{array}{l}\text { Sky clear blue after snow and hail of June } 6 . \\
\text { Press. aq. vap. }=1.98 \mathrm{~mm} \text {; temperature }=57^{\circ} \mathrm{F} \text { at } 11 \text { a.m. }\end{array}$ \\
\hline
\end{tabular}




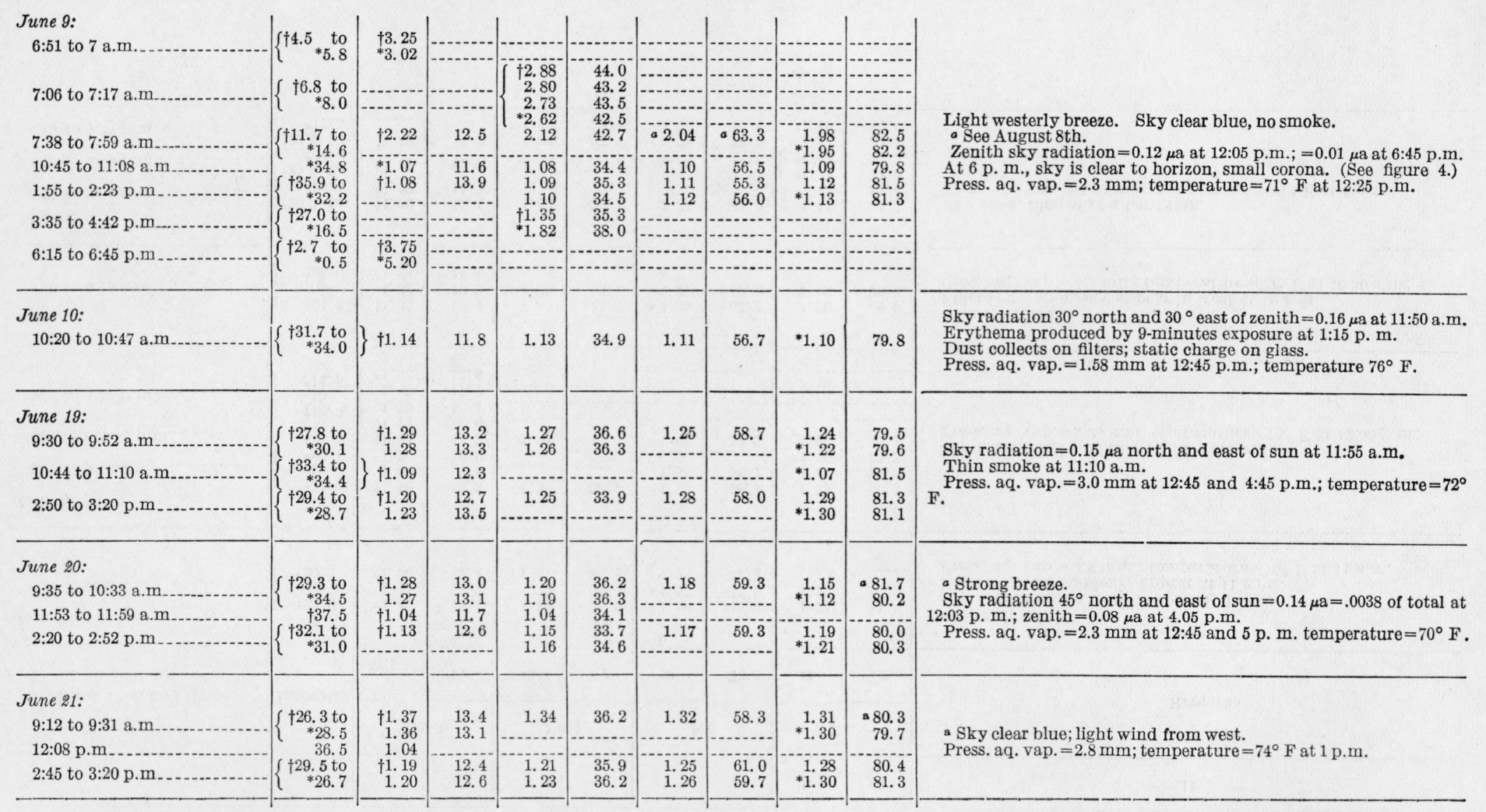


TABLE 1.-Filter transmissions of integrated ultraviolet solar radiation for different air masses, $m$, using a titanium photoelectric cell, Ti No. 4 (see fig. 3) - Continued

\begin{tabular}{|c|c|c|c|c|c|c|c|c|c|c|}
\hline 1 & 2 & 3 & 4 & 5 & 6 & 7 & 8 & 9 & 10 & 11 \\
\hline \multirow{2}{*}{ Date, 1934, and time } & \multirow{2}{*}{ Intensity } & \multicolumn{2}{|c|}{$\mathrm{Ba}-3$} & \multicolumn{2}{|c|}{$\mathrm{Ba}-1$} & \multicolumn{2}{|c|}{ Ni. } & \multicolumn{2}{|c|}{ Cx. } & \multirow{2}{*}{ Remarks } \\
\hline & & $m$ & $\mathrm{Tr}$ & $m$ & $\operatorname{Tr}$ & $m$ & $\operatorname{Tr}$ & $m$ & $\operatorname{Tr}$ & \\
\hline $\begin{array}{l}\text { June 22: } \\
\text { 8:51 to } 9: 58 \text { a.m }\end{array}$ & $\left\{\begin{array}{c}\mu \mathrm{a} \\
\dagger 23.0 \\
\text { to } \\
* 30.3\end{array}\right.$ & $\begin{array}{l}\dagger 1.47 \\
1.41 \\
* 1.20\end{array}$ & $\begin{array}{l}13.5 \\
13.3 \\
12.4\end{array}$ & $\begin{array}{l}\text { 1. } 39 \\
\text { 1. } 32 \\
1.31\end{array}$ & $\begin{array}{l}36.2 \\
35.6 \\
35.8\end{array}$ & $\begin{array}{l}\text { × } 1.29 \\
\text { a } 1.27 \\
1.25\end{array}$ & $\begin{array}{r}\text { a57.3 } \\
\text { a57.0 } \\
57.2\end{array}$ & $\begin{array}{r}1.24 \\
1.22 \\
\hdashline\end{array}$ & $\begin{array}{l}81.0 \\
80.7\end{array}$ & $\begin{array}{l}\text { a See July 6th. } \\
\text { Sky deep blue; no corona; westerly breeze. } \\
\text { Fracto-cumuli suddenly appear at } 11 \text { a.m. } \\
\text { Press. aq. vap. }=3.6 \mathrm{~mm} \text {.; temperature }=76^{\circ} \mathrm{F} \text { at } 11 \text { a.m. }\end{array}$ \\
\hline $\begin{array}{l}\text { June } 26: \\
\text { 8:55 to } 9: 40 \text { a.m. } \\
\text { 12:15 to } 12: 35 \text { p.m. } \\
\text { 4:32 to } 4: 58 \text { p.m. }\end{array}$ & $\left\{\begin{array}{c}\dagger 24.0 \\
\text { to } \\
* 30.4 \\
\dagger 37.8 \\
\dagger 18.5 \\
\text { to } \\
* 14.5\end{array}\right.$ & $\begin{array}{r}\dagger 1.46 \\
1.43 \\
* 1.26 \\
\dagger 1.04 \\
\left\{\begin{array}{r}\dagger 1.74 \\
1.79 \\
1.85 \\
* 1.95\end{array}\right.\end{array}$ & $\begin{array}{l}13.8 \\
13.8 \\
13.0 \\
11.7 \\
16.0 \\
16.4 \\
17.0 \\
16.8\end{array}$ & \begin{tabular}{r}
1.38 \\
\hdashline 1.04 \\
\hdashline$-1 .-$ \\
$-1 .-$
\end{tabular} & 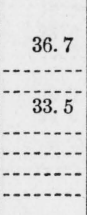 & $\begin{array}{r}1.37 \\
1.35 \\
\hdashline 1.04 \\
\hdashline-1.0 \\
\hdashline \\
\hdashline\end{array}$ & 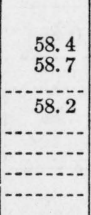 & 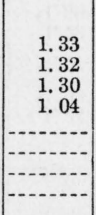 & 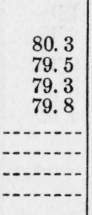 & $\begin{array}{l}\text { Sky blue; small corona. Light west wind. } \\
\text { Press. aq. vap. }=2.25 \mathrm{~mm} \text {; temperature }=76^{\circ} \mathrm{F} \text { at } 12: 50 \text { p.m. }\end{array}$ \\
\hline $\begin{array}{l}\text { July } 6: \\
\qquad 9: 16 \text { to } 9: 48 \text { a.m }\end{array}$ & $\left\{\begin{array}{c}\dagger 24.2 \\
\text { to } \\
* 28.0\end{array}\right.$ & $\left\{\begin{array}{l}+1.35 \\
1.33 \\
* 1.24\end{array}\right.$ & $\begin{array}{l}12.4 \\
12.3 \\
11.4\end{array}$ & $\begin{array}{r}1.32 \\
1.30 \\
-\end{array}$ & $\begin{array}{l}33.3 \\
33.1\end{array}$ & $\begin{array}{r}\text { a } 1.29 \\
\text { a } 1.27 \\
\end{array}$ & $\begin{array}{l}\text { a } 57.3 \\
\text { \& } 57.0 \\
-\end{array}$ & $\begin{array}{l}1.26 \\
1.25\end{array}$ & $\begin{array}{r}79.9 \\
79.5 \\
-\end{array}$ & $\begin{array}{l}\text { a See June } 22 \mathrm{~d} \text {. } \\
\text { Fracto-cirri suddenly appear in west at } 10 \mathrm{a} . \mathrm{m} \text {. } \\
\text { Press. aq. vap. }=5.3 \mathrm{~mm} \text {; temperature }=79^{\circ} \mathrm{F} \text { at } 10: 30 \mathrm{a} . \mathrm{m} \text {. }\end{array}$ \\
\hline $\begin{array}{l}\text { July 24: } \\
\text { 8:33 to 8:56 a.m } \\
\text { 3:22 to } 3: 53 \text { p.m } \\
\text { 4:07 to 4:38 p.m. }\end{array}$ & $\left\{\begin{array}{r}\uparrow 20.5 \text { to } \\
* 23.4 \\
\{25.5 \text { to } \\
* 24.3 \\
\begin{array}{r}\dagger 22.5 \text { to } \\
* 17.5\end{array}\end{array}\right.$ & $\begin{array}{l}\dagger 1.62 \\
* 1.47 \\
\dagger 1.37 \\
\dagger 1.57\end{array}$ & $\begin{array}{l}15.1 \\
14.2 \\
12.9 \\
14.6\end{array}$ & $\begin{array}{l}1.60 \\
1.40 \\
1.63 \\
1.69\end{array}$ & $\begin{array}{l}39.1 \\
35.7 \\
37.3 \\
36.9\end{array}$ & $\begin{array}{l}1.55 \\
1.44 \\
1.72 \\
1.75\end{array}$ & $\begin{array}{l}61.0 \\
58.3 \\
58.5 \\
57.8\end{array}$ & $\left\{\begin{array}{r}1.54 \\
1.53 \\
1.50 \\
*_{1} .53 \\
1.81 \\
*_{1.89}\end{array}\right.$ & $\begin{array}{l}81.7 \\
81.6 \\
81.3 \\
81.3 \\
81.7 \\
82.0\end{array}$ & $\begin{array}{l}\text { Sky clear, blue after a hard rain. } \\
\text { Press. aq. vap. }=4.34 \mathrm{~mm} \text {; temperature }=81^{\circ} \mathrm{F} \text { at } 5 \mathrm{p} . \mathrm{m} \text {. }\end{array}$ \\
\hline
\end{tabular}




\begin{tabular}{|c|c|c|c|c|c|c|c|c|c|c|}
\hline $\begin{array}{l}\text { July } 26: \\
\text { 12:50 p.m }\end{array}$ & 34.0 & 1.04 & 10.8 & & & & & & & $\begin{array}{l}\text { Ba-3 transmissions are low. High press. aq. vap. }=8.2 \mathrm{~mm} \text {; tem- } \\
\text { perature }=84^{\circ} \mathrm{F} \text { at } 1: 15 \text { p.m. }\end{array}$ \\
\hline $\begin{array}{l}\text { July } 27: \\
\text { 7:53 to } 8: 30 \text { a.m } \\
\text { 11:32 a.m }\end{array}$ & $\left\{\begin{array}{c}\dagger 11.4 \\
\text { to } \\
* 17.5 \\
29.8\end{array}\right.$ & $\begin{array}{r}\dagger 2.02 \\
1.98 \\
* 1.66 \\
1.06\end{array}$ & $\begin{array}{l}15.2 \\
14.8 \\
13.2 \\
10.9\end{array}$ & $\begin{array}{r}1.93 \\
1.91 \\
1.88\end{array}$ & $\begin{array}{r}39.0 \\
38.8 \\
38.6 \\
- \\
---\end{array}$ & $\begin{array}{l}1.84 \\
1.80 \\
1.78\end{array}$ & $\begin{array}{l}60.3 \\
60.2 \\
60.0\end{array}$ & $\begin{array}{r}1.76 \\
1.71 \\
-0\end{array}$ & $\begin{array}{r}81.3 \\
81.3 \\
-0\end{array}$ & $\begin{array}{l}\text { Sky white, wide corona. } \\
\text { Press. aq. vap. }=7.85 \mathrm{~mm} \text {; temperature }=81^{\circ} \mathrm{F} \text { at } 12: 30 \text { p.m. }\end{array}$ \\
\hline $\begin{array}{l}\text { August } 6 \text { : } \\
\text { 8:39 to } 9: 03 \text { a.m } \\
\text { 9:- } \\
\end{array}$ & $\left\{\begin{array}{l}\dagger 18.3 \\
\text { to } \\
* 22.2 \\
\dagger 27.1 \\
\text { to } \\
* 29.5\end{array}\right.$ & $\begin{array}{r}\dagger 1.64 \\
1.61 \\
1.52 \\
+1.30 \\
1.29 \\
*_{1} .23\end{array}$ & $\begin{array}{l}15.4 \\
14.5 \\
13.7 \\
13.2 \\
12.9 \\
12.8\end{array}$ & \begin{tabular}{r}
1.51 \\
$* 1.50$ \\
\hdashline 1.28 \\
\hdashline-2 \\
-2
\end{tabular} & \begin{tabular}{r}
36.3 \\
36.7 \\
\hdashline 36.4 \\
\hdashline-1 \\
-
\end{tabular} & $\begin{array}{r}1.27 \\
1.26\end{array}$ & $\begin{array}{l}58.9 \\
59.0\end{array}$ & $\begin{array}{r}1.25 \\
1.24 \\
-\end{array}$ & $\begin{array}{r}-8.2 \\
81.3 \\
80.8\end{array}$ & $\begin{array}{l}\text { Ti No. } 4 \text { values are nearly normal; sky is clearer than earlier. } \\
\text { Small corona after } 9: 40 \mathrm{a} . \mathrm{m} \text {. } \\
\text { Press. aq. vap. }=9.9 \mathrm{~mm} \text {; temperature }=71^{\circ} \mathrm{F} \text { at } 10: 50 \mathrm{a} . \mathrm{m} \text {. }\end{array}$ \\
\hline $\begin{array}{l}\text { August } 7: \\
\qquad 8: 28 \text { to } 9: 02 \text { a.m.-. }\end{array}$ & $\left\{\begin{array}{l}\left\{\begin{array}{l}\dagger 14.6 \\
\text { to } \\
* 19.5\end{array}\right. \\
\left\{\begin{array}{l}\dagger 24.3 \\
\text { to } \\
* 26.3\end{array}\right.\end{array}\right.$ & $\left\{\begin{array}{r}\dagger 1.74 \\
1.71 \\
1.54 \\
* 1.51 \\
\dagger 1.31 \\
1.30 \\
* 1.24\end{array}\right.$ & $\begin{array}{l}15.2 \\
14.9 \\
13.6 \\
13.2 \\
12.8 \\
13.0 \\
12.4\end{array}$ & \begin{tabular}{r}
1.68 \\
1.65 \\
\hdashline 1.29 \\
\hdashline-1. \\
-
\end{tabular} & $\begin{array}{r}40.2 \\
39.6 \\
35.6 \\
-0 \\
-0\end{array}$ & \begin{tabular}{r}
1.62 \\
1.61 \\
\hdashline 1.28 \\
1.27
\end{tabular} & \begin{tabular}{r}
61.3 \\
61.1 \\
\hdashline 58.6 \\
58.5 \\
-
\end{tabular} & \begin{tabular}{r}
1.58 \\
1.56 \\
\hdashline 1.26 \\
1.25
\end{tabular} & \begin{tabular}{r}
81.2 \\
81.8 \\
\hdashline 81.0 \\
81.0 \\
-0
\end{tabular} & $\begin{array}{l}\text { Sky clear, white around the sun. Light westerly breeze. } \\
\text { Fracto-cumulus clouds forming rapidly at } 10: 15 \text { a.m.; heavy rain } \\
\text { in p.m. } \\
\text { Press. aq. vap. }=9.44 \mathrm{~mm} \text {; temperature }=76^{\circ} \mathrm{F} \text { at } 10: 45 \text { a.m. }\end{array}$ \\
\hline $\begin{array}{l}\text { August 8: } \\
\text { 7:41 to 8:18 a.m }\end{array}$ & $\left\{\begin{array}{c}\text { t8. } \\
\text { to } \\
* 13.8 \\
\text { t33. } \\
\text { to } \\
* 34.5\end{array}\right.$ & $\begin{array}{r}2.30 \\
2.26 \\
2.22 \\
2.15 \\
1.94 \\
+1.10 \\
1.095 \\
* 1.09\end{array}$ & $\begin{array}{l}17.7 \\
18.3 \\
18.0 \\
17.8 \\
16.3 \\
12.6 \\
12.3 \\
12.1\end{array}$ & $\begin{array}{r}2.11 \\
2.08 \\
\hdashline-1.87 \\
\hdashline-1.8 \\
\hdashline- \\
\hdashline\end{array}$ & \begin{tabular}{r}
43.4 \\
43.2 \\
\hdashline-12.7 \\
-0 \\
-0
\end{tabular} & $\begin{array}{r}2.04 \\
2.01 \\
\hdashline\end{array}$ & 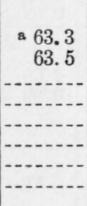 & $\begin{array}{r}\dagger 2.40 \\
2.36 \\
2.33 \\
1.99 \\
1.97 \\
\hdashline-1 .-\end{array}$ & \begin{tabular}{r}
83.0 \\
82.5 \\
82.4 \\
83.0 \\
83.5 \\
- \\
\hdashline.--- \\
.---
\end{tabular} & $\begin{array}{l}\text { Sky clear, small corona. At } 8: 05 \text { to } 8: 07 \text { a.m. ( } m=1.99 \text { to } 1.97 \text { ) } \\
\text { sky is whiter. } \\
\text { a See June } 9 \text { th. } \\
\text { At } 11: 20 \text { a.m., very faint haze (smoke) reduces the intensity by } \\
2 \text { to } 3 \text { percent. } \\
\text { Press. aq. vap. }=9.3 \mathrm{~mm} \text {; temperature }=75^{\circ} \mathrm{F} \text { at } 11: 50 \mathrm{a} . \mathrm{m} \text {. }\end{array}$ \\
\hline $\begin{array}{l}\text { August 17: } \\
\text { 8:09 to 8:38 a.m.- }\end{array}$ & $\begin{array}{l}\dagger 10.5 \\
\text { to } \\
* 14.9\end{array}$ & $\begin{array}{r}\dagger 2.03 \\
1.98 \\
* 1.74\end{array}$ & $\begin{array}{l}17.4 \\
17.0 \\
15.6\end{array}$ & $\begin{array}{l}1.96 \\
1.93\end{array}$ & $\begin{array}{r}42.0 \\
41.9 \\
-\end{array}$ & $\begin{array}{l}1.89 \\
1.87\end{array}$ & $\begin{array}{r}62.8 \\
62.7\end{array}$ & $\begin{array}{l}1.84 \\
1.81 \\
-1 .-\end{array}$ & $\begin{array}{r}81.5 \\
81.5 \\
-19-\end{array}$ & $\begin{array}{l}\text { Wide corona. Sky white; cirri on eastern horizon. } \\
\text { Cloudy at } 9: 30 \text { a.m. } \\
\text { Press. aq. vap. }=9.64 \mathrm{~mm} \text {; temperature }=74^{\circ} \mathrm{F} \text { at } 10: 15 \text { a.m. }\end{array}$ \\
\hline
\end{tabular}


TABLE 1.-Filter transmissions of integrated ultraviolet solar radiation for different air masses, $m$, using a titanium photoelecrtic cell, Ti No. 4 (see fig. 3 )-Continued

\begin{tabular}{|c|c|c|c|c|c|c|c|c|c|c|}
\hline 1 & 2 & 3 & 4 & 5 & 6 & 7 & 8 & 9 & 10 & 11 \\
\hline \multirow{2}{*}{ Date, 1934 , and time } & \multirow{2}{*}{ Intensity } & \multicolumn{2}{|c|}{$\mathrm{Ba}-3$} & \multicolumn{2}{|c|}{$\mathrm{Ba}-1$} & \multicolumn{2}{|c|}{ Ni. } & \multicolumn{2}{|c|}{ Cx. } & \multirow{2}{*}{ Remarks } \\
\hline & & $m$ & $\mathrm{Tr}$ & $m$ & $\operatorname{Tr}$ & $m$ & $\operatorname{Tr}$ & $m$ & $\mathrm{Tr}$ & \\
\hline $\begin{array}{l}\text { August 18: } \\
\text { 8:58 to } 9: 23 \text { a.m.-. }\end{array}$ & $\begin{array}{c} \\
\left\{\begin{array}{l}\mu \mathrm{a} \\
\dagger 17.9 \\
\text { to } \\
* 21.2\end{array}\right. \\
\left\{\begin{array}{l}+22.6 \\
\text { to } \\
{ }^{*} 25.6\end{array}\right.\end{array}$ & $\begin{array}{l}\left\{\begin{array}{r}\dagger 1.58 \\
1.57 \\
1.46 \\
*_{1.45} \\
\dagger 1.37 \\
1.36 \\
1.29 \\
1.28\end{array}\right.\end{array}$ & $\begin{array}{l}14.6 \\
14.1 \\
13.9 \\
13.1 \\
13.0 \\
12.9 \\
12.6 \\
12.3\end{array}$ & \begin{tabular}{r}
1.55 \\
1.52 \\
\hdashline 1.35 \\
1.34 \\
1.33 \\
\end{tabular} & \begin{tabular}{r}
38.3 \\
38.2 \\
\hdashline-15.7 \\
36.0 \\
35.9 \\
\end{tabular} & $\begin{array}{r}1.51 \\
1.49 \\
\hdashline 1.33 \\
1.32 \\
\\
\hdashline\end{array}$ & \begin{tabular}{r}
60.0 \\
60.0 \\
\hdashline 59.0 \\
58.8 \\
-0 \\
-0
\end{tabular} & \begin{tabular}{r|}
1.48 \\
1.47 \\
\hdashline 1.31 \\
1.30 \\
${ }^{*} 1.27$
\end{tabular} & \begin{tabular}{r}
80.9 \\
81.0 \\
\hdashline 81.7 \\
$\times 81.8$ \\
81.4
\end{tabular} & $\begin{array}{l}\text { Sky thick, white, clearing slowly, except eastern horizon. } \\
\text { "Washington sky." No breze. } \\
\text { a Sky seems whiter southeast of the sun at 10:01 a.m., and trans- } \\
\text { missions are higher. } \\
\text { Sky is bluer, clearer, small corona at } 9: 40 \text { to } 10: 10 \text { a.m. } \\
\text { Press. aq. vap. }=9.2 \text { mm; temperature }=79^{\circ} \mathrm{F} \text { at } 11: 10 \text { a.m. }\end{array}$ \\
\hline $\begin{array}{l}\text { August 23: } \\
\text { 8:31 to 8:59 a.m.-. }\end{array}$ & $\left\{\begin{array}{c}\dagger 12.9 \\
\text { to } \\
* 17.3\end{array}\right.$ & $\begin{array}{l}1.72 \\
1.70 \\
1.66\end{array}$ & $\begin{array}{l}\text { 14. } 7 \\
14.0 \\
14.0\end{array}$ & 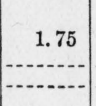 & 40.2 & $\begin{array}{r}1.80 \\
1.78 \\
\hdashline-1\end{array}$ & $\begin{array}{r}61.8 \\
61.6 \\
-\end{array}$ & $\begin{array}{r}\dagger 1.88 \\
1.84 \\
* 1.63\end{array}$ & $\begin{array}{l}83.7 \\
82.7 \\
81.3\end{array}$ & $\begin{array}{l}\text { Wide corona; sky pearly white; becoming completely hazy, with } \\
\text { fracto-cumulus at } 9: 45 \mathrm{a} . \mathrm{m} . \\
\text { Press. aq. vap. }=8.95 \mathrm{~mm} \text {; temperature }=71^{\circ} \mathrm{F} \text { at } 10 \mathrm{a} . \mathrm{m} \text {. }\end{array}$ \\
\hline $\begin{array}{l}\text { August 27: } \\
\text { 8:14 to 8:24 a.m.-. }\end{array}$ & $\left\{\begin{array}{l}\dagger 11.1 \text { to } \\
{ }^{*} 12.9\end{array}\right.$ & & & $\begin{array}{r}* 2.00 \\
\end{array}$ & 43.0 & $\begin{array}{l}2.05 \\
2.02\end{array}$ & $\begin{array}{l}63.3 \\
63.5\end{array}$ & $\begin{array}{r}\dagger 2.11 \\
2.08\end{array}$ & $\begin{array}{l}84.3 \\
83.9\end{array}$ & $\begin{array}{l}\text { Very unusual sky; thick overcast at } 6 \text { a.m.; clear except horizon, } \\
\text { from } 8 \text { to } 8: 24 \text { a.m.; suddenly overcast with fracto-cumulus clouds } \\
\text { at 8:27 a.m. } \\
\text { Press. aq. vap. }=10.2 \mathrm{~mm} \text {; temperature }=62^{\circ} \mathrm{F} \text { at } 8: 55 \text { a.m. }\end{array}$ \\
\hline $\begin{array}{l}\text { September } 1: \\
\text { 9:22 to } 9: 43 \text { a.m...- } \\
11: 27 \text { to } 11: 46 \text { a.m.-. }\end{array}$ & $\left\{\begin{array}{r}\dagger 21.1 \text { to } \\
* 24.3 \\
\dagger 31.8\end{array}\right.$ & $\begin{array}{r}\dagger 1.54 \\
* 1.38 \\
\dagger 1.15 \\
-\cdots\end{array}$ & \begin{tabular}{r|}
13.0 \\
12.4 \\
11.5 \\
-
\end{tabular} & $\begin{array}{l}1.49 \\
1.15 \\
1.14\end{array}$ & $\begin{array}{r}36.4 \\
333.3 \\
833.2\end{array}$ & 1.47 & \begin{tabular}{c|c}
59.3 \\
56.4
\end{tabular} & $\begin{array}{l}1.44 \\
1.42 \\
1.13 \\
-1 .--\end{array}$ & $\begin{array}{l}81.1 \\
81.3 \\
80.9 \\
-\end{array}$ & $\begin{array}{l}\text { - Fine cirrus clouds over the sun; reduce the intensity nonselec- } \\
\text { tively. } \\
\text { Sky clear after several days of rain. Corona small, pearly white. } \\
\text { Press. aq. vap. }=8.67 \mathrm{~mm} \text {; temperature }=72^{\circ} \mathrm{F} \text { at } 12: 15 \text { p.m. }\end{array}$ \\
\hline $\begin{array}{l}\text { September } 2 . \\
7: 20 \text { to } 7: 45 \text { a.m. }\end{array}$ & $\begin{array}{l}\text { †2.2 } \\
\text { to } \\
* 6.1\end{array}$ & $\left\{\begin{array}{r}\dagger 3.70 \\
3.53 \\
3.15 \\
3.10\end{array}\right.$ & $\begin{array}{l}20.5 \\
20.2 \\
19.7 \\
18.8\end{array}$ & $\begin{array}{r}3.25 \\
3.20 \\
\hdashline-1 .--\end{array}$ & 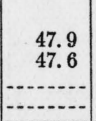 & $\begin{array}{r}3.35 \\
3.30 \\
3.27 \\
\hdashline-2 .\end{array}$ & $\begin{array}{r}66.7 \\
65.8 \\
65.5 \\
-\end{array}$ & $\begin{array}{r}3.68 \\
3.40 \\
\text { 3. } 05 \\
22.90\end{array}$ & $\begin{array}{l}83.8 \\
83.6 \\
83.0 \\
82.8\end{array}$ & $\begin{array}{l}\text { Sky clear at start. Observations stopped by sudden formation of } \\
\text { clouds. } \\
\text { Press. aq. vap. }=6.95 \mathrm{~mm} \text {; temperature }=72^{\circ} \mathrm{F} \text { at } 8 \mathrm{a} . \mathrm{m} \text {. }\end{array}$ \\
\hline
\end{tabular}




\begin{tabular}{|c|c|c|c|c|c|c|c|c|c|c|}
\hline $\begin{array}{l}\text { September } \$ \text { : } \\
\text { 12:05 to } 12: 30 \text { p.m........... }\end{array}$ & $\left\{\begin{array}{r}\dagger 23.5 \text { to } \\
{ }^{2} 22.6\end{array}\right.$ & $\begin{array}{l}\dagger 1.13 \\
* 1.12\end{array}$ & $\begin{array}{l}11.3 \\
11.1\end{array}$ & 1.13 & $\begin{array}{l}\text { a } 32.3 \\
\end{array}$ & 1.13 & 56.3 & 1.13 & 80.5 & $\begin{array}{l}\text { Remarkable day. Sky white; cloudless; smoky, hazy from brush } \\
\text { fire or perhaps smelters } 30 \text { miles south. } \\
\text { Zenith sky }=0.13 \text { to } 0.14 \mu \mathrm{a} ;=0.0058 \text { of total. (See June 20.) } \\
\text { Press. aq. vap. }=4.6 \mathrm{~mm} \text {; temperature }=75^{\circ} \mathrm{F} \text { at } 12: 50 \mathrm{p} . \mathrm{m} \text {. }\end{array}$ \\
\hline $\begin{array}{l}\text { September } 4: \\
8: 38 \text { to } 9: 07 \text { a.m.- } \\
11: 24 \text { to } 11: 58 \text { a.m.- }\end{array}$ & $\left\{\begin{array}{r}\dagger 10.8 \text { to } \\
* 12.6 \\
\dagger 29.1 \text { to } \\
* 29.6\end{array}\right.$ & $\begin{array}{r}+1.94 \\
1.73 \\
* 1.72 \\
+1.15 \\
* 1.13 \\
\end{array}$ & $\begin{array}{l}15.1 \\
14.3 \\
14.2 \\
10.8 \\
10.6\end{array}$ & $\begin{array}{r}1.87 \\
1.85 \\
1.14 \\
\hdashline-1 .-\end{array}$ & $\begin{array}{r}39.4 \\
39.2 \\
32.3 \\
-32\end{array}$ & $\begin{array}{l}1.83 \\
1.82 \\
1.14\end{array}$ & $\begin{array}{r}61.3 \\
60.8 \\
54.8\end{array}$ & $\begin{array}{l}1.79 \\
1.77 \\
1.13\end{array}$ & \begin{tabular}{r}
83.8 \\
83.3 \\
\hdashline 80.1 \\
-1
\end{tabular} & $\begin{array}{l}\text { Sky white; air "boils", but cannot see smoke. } \\
\text { s See September 3rd, same air mass. Short wave length ultra- } \\
\text { violet is greater than on September } 3 \text {. } \\
\text { Press. aq. vap. }=5.6 \mathrm{~mm} \text {; temperature }=76^{\circ} \mathrm{F} \text { at } 12: 20 \text { p.m. }\end{array}$ \\
\hline $\begin{array}{l}\text { September } 5: \\
\text { 7:37 to } 7: 56 \text { a.m.- } \\
\text { 8:56 to } 9: 18 \text { a.m. }\end{array}$ & $\left\{\begin{array}{r}+4.8 \text { to } \\
* 7.7 \\
+16.3 \text { to } \\
* 18.5\end{array}\right.$ & $\begin{array}{r}2.98 \\
2.95 \\
*_{2}^{2.70} \\
+1.80 \\
*_{1} .63\end{array}$ & $\begin{array}{l}19.1 \\
18.5 \\
18.1 \\
14.5 \\
14.1\end{array}$ & $\begin{array}{l}3.03 \\
2.98 \\
2.72 \\
1.77 \\
-\end{array}$ & $\begin{array}{l}46.0 \\
45.6 \\
44.5 \\
39.2\end{array}$ & $\begin{array}{l}3.17 \\
3.13 \\
2.77 \\
1.71\end{array}$ & $\begin{array}{l}66.7 \\
65.5 \\
64.7 \\
60.3\end{array}$ & $\begin{array}{r}\dagger 3.30 \\
2.86 \\
2.80 \\
1.69\end{array}$ & $\begin{array}{l}84.0 \\
82.3 \\
82.5 \\
80.7\end{array}$ & $\begin{array}{l}\text { Sky white, clear to horizon; wide corona. } \\
\text { Sky changing; billowy white streaks } 15^{\circ} \text { east of the sun. } \\
\text { Sometimes definite clouds. } \\
\text { Sky cloudy; thunderstorm on the San Francisco Peaks at } 10: 45 \\
\text { 8. m. } \\
\text { Press. aq. vap. }=8.8 \mathrm{~mm} \text {; temperature } 73^{\circ} \mathrm{F} \text { at } 9: 45 \text { a.m. }\end{array}$ \\
\hline $\begin{array}{l}\text { September } 7: \\
\text { 8:15 to } 8: 37 \text { a.m...- } \\
\text { 10:05 to } 10: 28 \text { a.m.- } \\
\text { 10:45 to } 11: 02 \text { a.m.- }\end{array}$ & $\left\{\begin{array}{r}\dagger 10.3 \text { to } \\
*_{13.2} \\
\dagger 25.2 \text { to } \\
*_{227.4} \\
\dagger 28.7 \text { to } \\
* 30.0\end{array}\right.$ & $\begin{array}{r}2.10 \\
2.07 \\
\dagger 1.38 \\
1.33 \\
\dagger 1.26 \\
*_{1.23}\end{array}$ & $\begin{array}{l}16.8 \\
16.3 \\
13.5 \\
13.0 \\
12.6 \\
12.3\end{array}$ & $\begin{array}{r}2.14 \\
1.36 \\
* 1.31 \\
1.25\end{array}$ & \begin{tabular}{l}
42.7 \\
\hdashline 36.4 \\
35.6 \\
34.9
\end{tabular} & $\begin{array}{r}2.22 \\
2.19 \\
1.35 \\
1.24\end{array}$ & $\begin{array}{r}63.5 \\
63.3 \\
58.9 \\
\hdashline 57.8\end{array}$ & $\begin{aligned} \dagger 2.28 \\
* 2.04 \\
1.34\end{aligned}$ & $\begin{array}{r}83.8 \\
82.7 \\
81.5 \\
\hdashline 80.2\end{array}$ & $\begin{array}{l}\text { Sky clear to horizon; corona, pearly white; light, southwesterly } \\
\text { breze. } \\
\text { Corona narrower; sky clear blue; fracto-cumulus on San Francisco } \\
\text { Peaks. } \\
\text { Sky turning fracto-cumulus clouds at } 11: 15 \text { a.m.; thunder over the } \\
\text { Elden Mountain. } \\
\text { Sky radiation: Zenith }=0.1 \mu \text { a; angle } 45^{\circ}, \mathrm{S} . \mathrm{W} .=0.12 \mu \mathrm{a} \text { at } 10: 40 \\
\text { a.m. } \\
\text { Press. aq. vap. }=6.8 \mathrm{~mm} \text {; temperature }=74^{\circ} \mathrm{F} \text { at } 11: 25 \text { a.m. }\end{array}$ \\
\hline 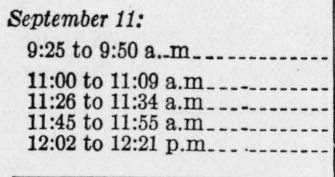 & $\left\{\begin{array}{r}\dagger 23.0 \text { to } \\
* 25.5 \\
+32.3 \\
* 34.2 \\
* 34.8 \\
* 35.4\end{array}\right.$ & $\begin{array}{l}1.55 \\
* 1.50 \\
+1.25 \\
* 1.20 \\
1.18 \\
* 1.16\end{array}$ & $\begin{array}{l}13.6 \\
13.6 \\
12.3 \\
12.0 \\
11.9 \\
11.9\end{array}$ & $\begin{array}{r}1.59 \\
\hdashline 1.16\end{array}$ & \begin{tabular}{|r|}
37.6 \\
\hdashline 34.7 \\
\hdashline
\end{tabular} & \begin{tabular}{|c|}
1.61 \\
\hdashline 1.16
\end{tabular} & \begin{tabular}{|c|}
59.8 \\
\hdashline-1 \\
-57.3 \\
\end{tabular} & $\begin{array}{r}\dagger 1.63 \\
1.52 \\
1.23 \\
1.20 \\
* 1.17 \\
1.16\end{array}$ & $\begin{array}{l}81.7 \\
81.7 \\
81.1 \\
80.4 \\
80.5 \\
80.3\end{array}$ & $\begin{array}{l}\text { Sky clear, blue; small corona; light westerly breeze. } \\
\text { Few clouds on eastern horizon at } 9: 52 \text { a. } \mathrm{m} \text {. } \\
\text { Press. aq. vap. }=5.4 \mathrm{~mm} \text {; temperature }=73^{\circ} \mathrm{F} \text { at } 1 \text { p.m. }\end{array}$ \\
\hline $\begin{array}{l}\text { Septcmber 12: } \\
\text { 8:05 to 8:31 a.m }\end{array}$ & $\left\{\begin{array}{r}t 10.1 \\
\text { to } \\
* 14.3 \\
\left\{\begin{array}{r}\dagger 23.3 \text { to } \\
* 27.5 \\
40.3\end{array}\right.\end{array}\right.$ & $\begin{array}{l}+1.67 \\
* 1.47 \\
-\end{array}$ & $\begin{array}{r}12.4 \\
11.8 \\
\end{array}$ & \begin{tabular}{r}
1.61 \\
\hdashline \\
\end{tabular} & \begin{tabular}{r|r}
33.9 \\
\hdashline-10 \\
\end{tabular} & \begin{tabular}{r}
2.51 \\
2.46 \\
$-{ }^{*} .19$ \\
1.59 \\
\hdashline-1.2 \\
\end{tabular} & \begin{tabular}{r}
64.3 \\
63.4 \\
62.5 \\
58.6 \\
\hdashline$-1 .-$ \\
\end{tabular} & $\begin{array}{r}\dagger 2.65 \\
2.54 \\
2.24 \\
1.54 \\
1.52 \\
1.19 \\
\end{array}$ & $\begin{array}{l}81.5 \\
81.3 \\
80.5 \\
\end{array}$ & $\begin{array}{l}\text { Clouds low on eastern horizon at } 8 \mathrm{a} . \mathrm{m} \text {. Sky deep blue, clearer } \\
\text { than yesterday; very small or no corona. Light southwesterly } \\
\text { breeze. Clearer than September } 7 \text {; lower transmissions for larger } \\
\text { air mass. } \\
\text { Press. aq. vap. }=5.3 \mathrm{~mm} \text {; temperature }=73^{\circ} \mathrm{F} \text { at } 12: 25 \mathrm{p} . \mathrm{m} \text {. }\end{array}$ \\
\hline
\end{tabular}


TABLE 1.-Filter transmissions of integrated ultraviolet solar radiation for different air masses, $m$, using a titanium photoelectric cell, Ti No. 4 (See fig. 3)-Continued

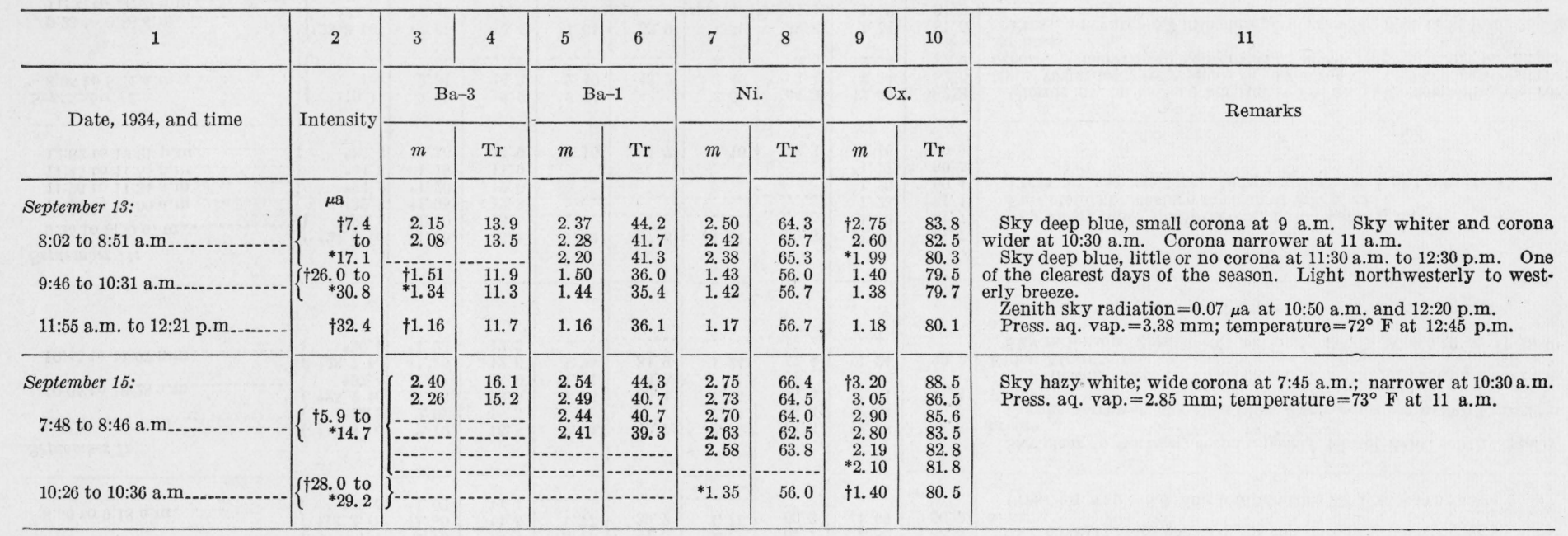




\section{CLIMATIC CONDITIONS AT THE OBSERVING STATIONS ${ }^{3}$}

During the past year an opportunity was presented to supplement the measurements in progress in Washington (Lat. $38^{\circ} 56^{\prime} \mathrm{N}$; elevation $350 \mathrm{ft}$ ) with similar measurements at the Lowell Observatory, Flagstaff, Ariz. (Lat. $35^{\circ} 12^{\prime} \mathrm{N}$; elevation $7,300 \mathrm{ft}$ ); also on the nearby San Francisco Peaks, at an elevation of 10,500 ft; and at the School of Tropical Medicine, San Juan, P. R. (Lat. $18^{\circ} 28^{\prime}$ N; elevation about $75 \mathrm{ft}$ ).

At the Flagstaff station successful measurements of ultraviolet solar radiation were obtained under various conditions; high and low humidity, large and small air masses, clear and smoky sky, etc. The data obtained at San Juan are of interest in connection with the question of ultraviolet solar intensities in the tropics.

Incidentally, it is of interest to record climatic conditions during the observations at the three stations. The measurements at Flagstaff, Ariz., were made during the last half of May to the middle of September 1934. This includes a relatively dry, rainless season (May-June) with noon-hour temperatures ranging from $55^{\circ} \mathrm{F}$ to $75^{\circ} \mathrm{F}$, and with pressures of aqueous vapor ranging from 1.6 to $3.6 \mathrm{~mm}$; and a so-called "rainy season" (during July and August), with noon temperatures up to $85^{\circ} \mathrm{F}$ and pressures of aqueous vapor ranging from 5 to $10 \mathrm{~mm}$.

Weather conditions during the rainy season were fairly regular in sequence, with clear sky during the night and usually until noon or midafternoon, followed by cloudiness (and sometimes rain) clearing again early in the evening, when the cycle was repeated. While this is disconcerting to the meteorologist, it is a boon to the astronomer.

At the Flagstaff station when the wind was from the northwest, the sky was free from smoke. However, when there was a light breeze, the observations were sometimes affected by an almost invisible blue wood smoke (closely matching the blue sky) from a sawmill situated several miles to the south, or by visually imperceptible coal smoke (see fig. 4) from a transcontinental train, some 30 miles to the west. Since the latter disturbance occurred at certain times of the day, no measurements were made at those times.

The ultraviolet measurements at San Juan were made during February and the first half of March 1935. During this time, the forenoon measurements were often interrupted by alternate cloudiness (rapidly moving fracto-cumulus) and sunshine. By noon, and often continuing throughout the entire afternoon, the whole sky was cloudless,- except along the south and southwest horizon, over the inland mountains, where the morning clouds (now cumulus and stratocumulus) had been blown by the prevailing north and northeast winds.

The School of Tropical Medicine being situated on the north shore of the island, the observing station was free from smoke from ships in the harbor, close by to the south, and from burning refuse of harvested sugarcane on the plantations still farther to the south.

\footnotetext{
3 The observations of the effect of altitude at Flagstaff, Ariz., were obtained with grants from the council on Physical Therapy of the American Medical Association and from the American Academy of $/$ rts and Sciences. The measurements on the effect of latitude were obtained in connection with the standardization of ultraviolet meters at the School of Tropical Medicine, San Juan, P. R., under the auspices of Columbia University.
} 
While there are numerous, perfectly cloudless days, which occur at irregular intervals during the year, no characteristic, long-continued, cyclical, regularly recurring, clear-weather conditions can be described for the Washington station. Situated on high ground in the northwest part of the city, and gradually being surrounded by residences, high-precision ultraviolet observations for standardization purposes are made only on the clearest days. These usually occur when the wind is from the northwest or west, especially after a hard rain, when the air over the southeastern part of the city (through which the forenoon measurements must be made, especially during the winter months) is relatively free from pollution by smoke and dust. The ultraviolet measurements made under various meteorological conditions are of statistical interest indicating the amount of ultraviolet available in a city relatively free from factory smoke.

\section{MINOR OBSERVATIONS}

In addition to the prolonged measurements of the effect of solar altitude (air mass), and of the geographic latitude and the altitude of the observing station, similar observations, of short duration, were made of the effect of numerous local meteorological conditions upon the spectral quality and total intensity of ultraviolet solar radiation

For example, the effect of smoke from burning coal or wood is to reduce the total intensity, and to increase the percentage transmission of the filters, indicating a highly selective absorption of the short wavelength ultraviolet solar rays. That this is not always true is shown by measurements through a peculiar thin smoke of undetermined origin (whether of forest fire or smelters) that suddenly enveloped the Flagstaff station for a day (September 3, 1934, sky cloudless, white, no distinct corona). While this reduced the total ultraviolet intensity by almost 35 percent (see fig. 4) the transmissions of ultraviolet through the filters remained unchanged and in agreement with the values prevailing at that season, suggesting a uniform ("gray") absorption throughout this spectral region.

At unexpected times (July 30, 1934; a hot day; ozone should be low) the filter transmissions were lower (the $\mathrm{Ba}-3$ filter transmissions remained fairly constant, amounting to 11.3 to 11.5 percent, between $9: 30$ and 10 a. $m .-m=1.30$ to $m=1.23$ ) than the prevailing values for a given air mass, observed at the Flagstaff station at that season of the year. This could not be accounted for on the basis of observational errors or unclean filters. A more plausible explanation is that there was either an increase in atmospheric transparency, caused by a temporary decrease in the amount of ozone in the stratosphere, or a temporary increase in emission of short wavelength ultraviolet solar radiation. Similarly, as will be more fully described on a subsequent page, on January 24,1935 , which was an exceedingly clear day in Washington, the filter transmissions were unusually high (fig. 7) whereas, on the basis of a clear sky, free from local atmospheric pollution, there should have been an increase in intensity of ultraviolet, of the shortest wave lengths, hence the filter transmissions should have been lower. Apparently, the high transmissions were owing to an unseasonably high ozone content (equivalent to a layer of the pure gas, $3 \mathrm{~mm}$ in thickness at normal temperature and pressure, ntp; see fig. 7). 
Thin, cirrus clouds over the sun which reduced the intensity by several percent, did not appreciably affect the spectral qualities indicated by the filter transmissions. The effect of high humidity (as indicated by a pearly white corona) on ultraviolet absorption is not marked through a small air mass, but is observable through a large air mass.

The sea-level observations indicate that the short wave length ultraviolet solar spectral energy curve is constantly changing as the result of variations in atmospheric pollution. Hence, where great refinements are required, no standard ultraviolet solar spectral energy curve can be assumed for a given air mass.

On exceptionally clear days in Washington, and especially at the higher elevations, the measurements are consistent in showing a higher atmospheric transparency to ultraviolet, for the same solar altitudes, in the afternoon than in the forenoon (fig. 5), which is explained on the basis of a decreased ozone content, principally by thermal decomposition (4).

In agreement with expectations, the transmission of ultraviolet solar radiation through the various filters increases with increase in air mass. At the Flagstaff station atmospheric conditions were so constant (see fig. 4) that a definite change in transmission was observable in the course of a few minutes, corresponding with a small change in air mass, amounting to only $\Delta m=0.02$.

\section{FOG}

Interesting ultraviolet measurements were made through a light fog of short duration in Washington, at 2:30 p. m., January 21, 1935. When the fog was fairly homogeneous, the sun's disk appeared white, and the integrated ultraviolet intensities were less than 10 percent as great as observed during clear weather through a similar air mass, $m=2.5$. When the fog began to form into clouds the ultraviolet intensities increased to one-fifth the clear weather values. In both cases, within the errors of observation, the average values of the filter transmissions were closely the same as observed during clear skies, with the sun shining through the same air mass, indicating that the fog particles (water vapor) exerted but little selective absorption upon the transmitted ultraviolet rays.

\section{SNOW}

It is well known that snow is a strong reflector of ultraviolet rays. Measurements (5) on July 28, 1932, on the Jungfraujoch, (elevation $11,350 \mathrm{ft}$ ), using a wide-angle receiver indicated that the ultraviolet reflected from snow was almost as intense as that of direct solar radiation.

The Jungfraujoch observing station is situated on one mountain side and faces a similar, steeply inclined, close-by mountain side, with the intervening valley covered with glacial ice and snow. Hence it is an excellent location for the measurement of ultraviolet sky and direct solar radiation, through light fog and falling snow. All measurements (5) show a high intensity of ultraviolet through fine mist and snow particles. 


\section{SKY}

Ultraviolet radiation from the sky is indefinable without a specification of atmospheric conditions. It has been mentioned repeatedly by other observers. Using a wide-angled receiver, on the Jungfraujoch (5) with the sun obscured by mist or fine particles of falling snow, the ultraviolet intensity was almost one-half that of direct sunlight, in a cloudless sky. At the Flagstaff station, using an aperture of $22^{\circ}$, with a cloudless, deep-blue sky, showing practically no corona, the ultraviolet sky radiation at an angle of $45^{\circ}$ from the sun at the noon hour, was only 0.38 percent as great as direct solar radiation. When the atmosphere was hazy, smoky (e. g., September 3, above mentioned, when the direct ultraviolet solar intensity was reduced by one-third) the sky radiation was 0.58 percent as great as direct ultraviolet solar radiation, or 65 percent greater than during: the clearest weather. (See table 1, data for June 20 and September 3, 1934.)

Sky radiation is most pronounced at sea-level stations. Using a receiver with a wide aperture $\left(45^{\circ}\right)$ at Washington, (6) the ultraviolet sky radiation in a $45^{\circ}$ angle directly surrounding the sun, amounted to about 7 percent of the total incident solar ultraviolet, under average atmospheric conditions; rising to 19 percent of the total measured when the sky was turbid from a forest fire, the smoke of which reduced the intensity of the directly incident solar ultraviolet to almost one-third the value observed through a clear sky.

Atmospheric pollution by dust, transported long distances, as experienced in Washington in March-April 1934 and 1935, appears to have only a very temporary effect upon ultraviolet transmission and is entirely different from the prolonged effect of dust that is blown to high altitudes, by volcanic eruptions, such as, for example, the eruption of Krakatoa, which is often cited with reference to the effect of atmospheric dust upon meteorological conditions.

For example, the ultraviolet solar intensities, for $m=1.20$, as measured with the standard ultraviolet meter (using Ti No. 4) on May 7, 1934, and May 18, 1935, differed by less than 6 percent; also, the transmissions of the filters (Tr. of $\mathrm{Cx} .-\mathrm{D}=80.8$ and 81.1 percent, respectively) showed no difference in the spectral quality of the solar ultraviolet on these two dates, from which it appears that the dust had been removed by heavy rains. This, of course, was apparent also from visual observations of atmospheric turbidity.

\section{ERYTHEMA TESTS}

In a previous communication (17) data were given on the time of exposure (the total ultraviolet solar energy) required to produce a minimum perceptible erythema. In that paper it was shown that an exposure of 20 to 30 minutes to a midsummer noonday sun is required to produce a minimum perceptible erythema. It was also shown that to produce a minimum perceptible erythema, in the winter when the skin is dry, about twice as much energy (twice the time of exposure using standard wave length $2967 \mathrm{~A}$ ) is required as in the summer, when the skin is moist.

In the above-mentioned tests (17), before exposure to heterogeneous ultraviolet radiation the skin-sensibility was standardized by exposure 
to homogeneous radiation of wave length $2967 \mathrm{~A}$, of known energy value. Such a preliminary standardization was not practicable before making the erythema tests outside of Washington. Hence only general statements regarding erythemogenic action can be made.

The erythema test at the Lowell Observatory station (elevation $7,300 \mathrm{ft}$ ) was made early in the afternoon (1:08 to 1:22 p.m.; air mass, $m=1.04$ to 1.08 ) on a cloudless day (June 10,1934). The temperature was $76^{\circ} \mathrm{F}$, and the vapor pressure was down to $1.58 \mathrm{~mm}$. It is therefore presumed that the skin was dry, requiring a longer exposure to produce a minimum perceptible erythema than if the skin had been moist from perspiration, which occurs during exposure to the sun.

Small areas of the left upper inside arm were exposed for intervals of $2,5,7,9,10,12$, and 14 minutes, respectively. The exposures of 12 and 14 minutes, respectively, evidently were too long-the skin being sensitive to the clothing worn. On the evening of June 11, the four longest exposures appeared lightly pigmented; and a week later (June 17) they were still visible being pigmented dark brown. The time of exposure to produce a minimum perceptible erythema appeared to be between 9 and 10 minutes or about one-third (to one-half) the time for a low-level exposure.

During the erythema tests at the San Juan station the exposures (made through an air mass $m=1.1$, March 11, 1935) were interrupted by passing clouds. A minimum perceptible erythema was produced by an exposure of 21 to 22 minutes, which is not markedly shorter than observed on a moist skin, exposed at Washington. The most important observation was that the shortest exposures (of 14 and 18 minutes; utilizing about 450,000 to 600,000 ergs of ultraviolet radiation) showed no erythema, but after several days showed slight pigmentation which continued to be visible for several weeks. To what extent this is caused by the humid sea air is unknown.

It is important to note that tanning is caused not only by wave lengths (including, and shorter than, $3130 \mathrm{~A}$ ) that produce an erythema but also by wave lengths between 3200 to $3300 \mathrm{~A}$ that have little or no power to produce an erythema; also that the tanning resulting from long exposure at the San Juan station was a characteristic light brown, as distinguished from the dark brown obtained at the Flagstaff and Washington stations.

The present measurements substantiate the earlier data (5) showing that the greater susceptibility to sunburn at the seashore is not explainable exclusively on the basis of an excessively higher ultraviolet radiation than obtains at the same level inland; but that skin sensitization by sea water, and increased penetrability of the rays into the moist skin must also be considered.

\section{EFFECT OF ALTITUDE ON ULTRAVIOLET SOLAR RADIATION}

As already mentioned, by using a selective radiometer that responds only to the ultraviolet of wave lengths shorter than about $3300 \mathrm{~A}$, and a group of 4 glass filters, it is possible to analyze the everchanging spectral quality and total intensity of radiation in this narrow band (about $400 \mathrm{~A}$ in width) of ultraviolet in sunlight, apparently with as high degree of reliability as anything yet attained by means of spectral radiometry. 
The results of these measurements corroborate what was already known in a general way, that ozone, which has a high absorption in the ultraviolet (2200 to $3200 \mathrm{~A}$ ) and is situated in greatest abundance in the stratosphere, at an average height of 21 to $24 \mathrm{~km}$, depending upon the station (9), (10), (11), determines the spectral quality of ultraviolet radiation incident upon the earth's surface. Water, in the vapor state, as it ordinarily exists in the atmosphere, and as observed in a light fog, appears to be relatively nonselective in spectral ultraviolet transmission, the loss in intensity being caused by scattering, principally by reflection within the particles of water.

The amount of ultraviolet reaching the earth's surface is controlled by ozone in the stratosphere and by atmospheric pollution, by dust and smoke, near the surface. The chief gain in ultraviolet intensity and, to a less degree, in spectral quality, appears to result from rising above the first $7,000 \mathrm{ft}(2 \mathrm{~km})$ elevation above sea level, in which occurs local pollution by smoke and dust. To eliminate a large amount of the ultraviolet absorption by ozone would require an elevation so great that it would be impracticable for large-scale physiological and biological tests.

By means of ultraviolet filter transmission measurements, at high elevations, it is possible to observe small variations in the transparency of the upper atmosphere that are masked or rendered doubtful by rapid changes in local pollution when similar measurements are made at low elevations.

Outstanding among the measurements made at Flagstaff, Ariz., is the observation of a lower filter transmission, indicating a higher atmospheric transparency and a consequent greater amount of incident ultraviolet of the shortest wave lengths, for the same air mass, in the afternoon than in the forenoon (fig. 5). A good example is a set of measurements made on June 26, 1934, using Ti No. 1 photoelectric cell and the Ba.-3 filter. At 9:53 a.m. $(m=1.21)$ the transmission of this filter was 25.7 percent and at $2: 54$ p.m. $(m=1.21)$ it transmitted 24.8 percent.

This greater atmospheric transparency in the afternoon than in the forenoon was especially noticeable during the dry season, in MayJune, with the sky cloudless to the horizon throughout the day (and night) when, judged by the size of the corona (if any was visible) surrounding the sun, there appeared to be no visual difference in (a.m. and p.m.) atmospheric conditions, for the same solar altitude. The marked difference in solar altitude for the same filter transmission (e. g., air mass $m=1.25$ in the a.m. and $m=1.40$ in the p.m. in June) seems sufficient to be unmistakable in indicating a greater atmospheric transparency in the afternoon. Similarly the observations show a higher transparency in the autumn than in the spring-a phenomenon previously observed by others (7), (8), by means of spectrographic measurements of ultraviolet intensities at different solar altitudes.

In this connection it is to be noted that pollution of the air by smoke (from the above-noted railroad engine) in the afternoon, would have selectively absorbed the ultraviolet of short wavelengths and, hence, increased the filter transmissions. This difference in atmospheric transparency from forenoon to afternoon is, therefore, to be ascribed to variations in the amount of ozone in the stratosphere. From the 
data given in figures 5 and 7 it is estimated that the observed decrease in ozone amounted to $0.1 \mathrm{~mm}$ (ntp).

An explanation, by Kidson (4), to account for the presence and variability of ozone, is based primarily upon a photochemical reaction caused by short wave length ultraviolet solar radiation. Among other causes, radiation of wave lengths shorter than $1850 \mathrm{~A}$, acting upon oxygen in the uppermost atmosphere, produces ozone which in turn is subjected to decomposition by heat and by solar radiation of wave lengths $2200 \mathrm{~A}$ to $3300 \mathrm{~A}$. In this manner an equilibrium in photochemical action is established, which is a function of the ultraviolet solar intensity, and the temperature of the upper atmosphere.

This accounts for the relatively constant amount of ozone in the tropics and the annual variations in the higher latitudes, where the amount of ozone is a maximum in the local spring and a minimum in

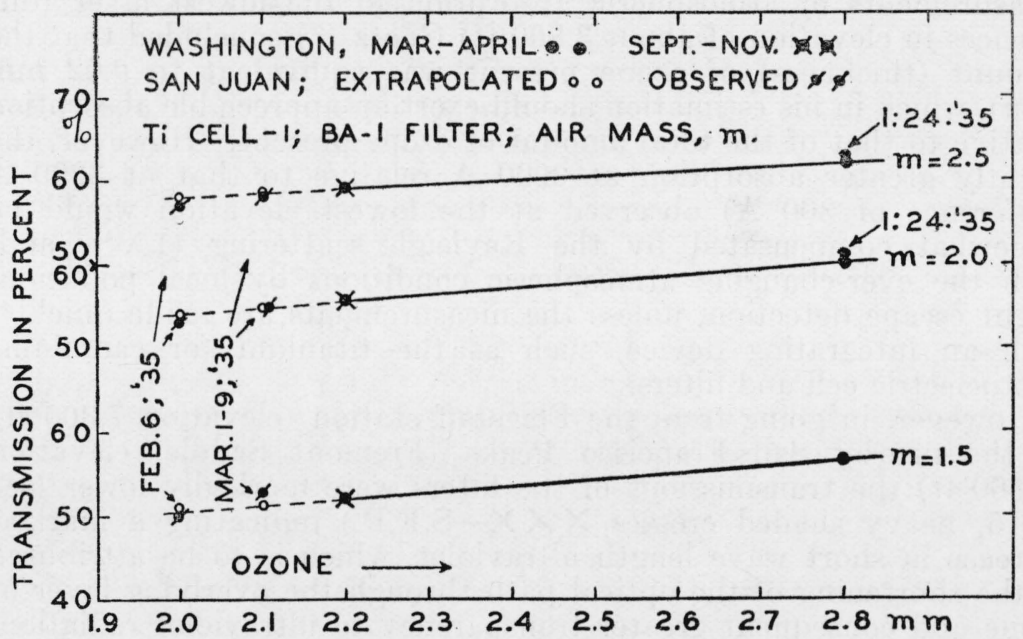

Fig. 7.-Filter transmissions of ultraviolet solar radiation after passing through various air masses containing different amounts of ozone.

the local autumn. In addition to these variations, Dobson and Meetham (12) report marked daily variations in the total amount of ozone (in Europe) with varying meteorological conditions; the maximum amounts being generally observed with cyclones and the reverse with anticyclones. In view of these facts a more complete study of the data obtained at Flagstaff in July 1934, in connection with the barometric pressures at that time may perhaps disclose the reason for the low filter transmissions (apparent low ozone content and high ultraviolet intensities) observed at that time.

From the measurements obtained with the titanium photoelectric cells and filters, and from the earlier, less extensive spectral-energy data, it appears that, for a given solar altitude (say air mass, $m=2.0$ ), at sea level (Washington) the relative spectral-energy distribution, of the solar radiation shining through an atmosphere free from local pollution, is closely the same (at least in the small spectral range of 2900 to $3300 \mathrm{~A}$ ) as that observed at a much higher elevation (Flagstaff, Ariz., altitude 7,300 ft) for an appreciably lower solar altitude (larger apparent local air mass, $m=2.4$ ). 
The spectral-energy data obtained with a spectroradiometer, by Greider and Downs (13) at two stations differing widely in altitude (at Springfield Lake, Ohio and Colorado Springs, Colo.) support these observations showing that, while the total intensities differ appreciably, within the experimental errors in making the observations, there is no marked difference in the shape of the spectral-energy distribution in this narrow spectral range for equivalent air masses, at these two stations.

The foregoing observations are to be expected, owing to the fact that the shape of the part of the solar spectral-energy curve in the region of $2900 \mathrm{~A}$ to $3200 \mathrm{~A}$ is determined largely by a layer of ozone, which has a strong absorption band that extends from about 2200 A to $3200 \mathrm{~A}$, with a maximum at about $2530 \mathrm{~A}$. The average amount of ozone ${ }^{4}$ in this layer is equivalent to about $3 \mathrm{~mm}$ (ntp). From his measurements of atmospheric absorption in the lowest layer (differences in elevation of about $3,800 \mathrm{ft}$ ) Goetz (7) concluded that the amount (thickness) of ozone present was equivalent to $0.02 \mathrm{~mm}$ (ntp), which in his estimation should exert an appreciable absorption relative to that of the total amount of ozone present. However, the slightly greater absorption at $2900 \mathrm{~A}$ relative to that at $3200 \mathrm{~A}$ (difference of $300 \mathrm{~A}$ ) observed at the lowest elevation would be somewhat compensated by the Rayleigh scattering $\left(1 / \lambda^{4}\right)$, and, with the ever-changing atmospheric conditions by local pollution, might escape detection, unless the measurements are made quickly, with an integrating device, such as the titanium (or cadmium) photoelectric cell and filters.

However, in going from the Flagstaff station (elevation $7,300 \mathrm{ft}$ ) to the nearby San Francisco Peaks (Fremont Saddle, elevation $10,500 \mathrm{ft}$ ) the transmissions of the filters were markedly lower (see fig. 5, heavy shaded crosses $\times X \times-$ S.F.P.) indicating a marked increase in short wave length ultraviolet, which is to be attributed to the shortening of the optical path through the overlying layer of ozone and consequent greater transparency to ultraviolet radiation. Measurements at the higher elevation (Fremont Saddle, elevation $10,500 \mathrm{ft}$ ) were made during midday on June 12 and July 5, 1934, under fairly clear atmospheric conditions. The total ultraviolet intensity, as measured with Ti No. 4, was 18 percent higher on June 12 , and 15 percent higher on July 5 , than observed under similar conditions at the Lowell Observatory station (elevation 7,300 ft).

Further evidence of greater atmospheric transparency in the fall as compared with early summer, is shown in figure 4, where the intensities for June 7 and September 11 , for air mass $m=1.25$ are connected with a dotted line. The value for September 11 is about 7 percent higher-4.5 percent higher after correction for difference in solar distance. The intensities at the noon hour, on those two dates are closely the same in spite of the differences in solar altitude $-m=1.04$ and 1.16 , respectively. It is relevant to add, that the air masses (solar altitudes) are so closely the same during the first part of June that no attempt was made to plot the intensities relative to solar altitude instead of the time of the day.

${ }^{4}$ Dobson (8) found that over continental Europe the atmospheric ozone varies in a seasonal cycle rang. ing from a maximum of about $3.3 \mathrm{~mm}$ (ntp) in April to $2.3 \mathrm{~mm}$ in October. Goetz cites variations between $1.7 \mathrm{~mm}$ and $4.2 \mathrm{~mm}$. Regner (9) found 70 percent of the ozone below $30 \mathrm{~km}$. 


\section{EFFECT OF GEOGRAPHIC LATITUDE ON ULTRA- VIOLET SOLAR RADIATION}

Measurements of ultraviolet solar radiation intensities in the tropics, particularly near the seashore, are of interest because of a vaguely defined belief that the sea air imparts some peculiar physiological property to these rays.

The intensity of the ultraviolet rays reaching the earth's surface is determined principally by the amount absorbed by the ozone in the stratosphere, and only to a minor extent by absorption in the lower atmosphere. The lower stratum of air, over the ocean, cannot restore the ultraviolet absorbed in the upper atmosphere. The higher ultraviolet intensity, if any, at the seashore, as compared with an inland sea-level station, is owing to a greater atmospheric transparency, caused by a greater freedom from dust and smoke, which is determined by the direction of the wind.

This question was discussed in an earlier paper (5) in which it was shown that the ultraviolet radiation intensities observed over the ocean were not conspicuously higher than at a sea-level station, situated inland, at the same latitude and at the same season of the year. Recently, in connection with the other radiometric work at San Juan, P. R., an opportunity was presented to verify these earlier measurements, using greater refinements in making the observations.

The ultraviolet measurements, made in San Juan (Lat. $18^{\circ} 28^{\prime}$ N.) in February and the first half of March 1935, were preceded and followed by similar measurements, made respectively during the latter part of January, and the latter part of March 1935, at Washington, D. C. (Lat. $38^{\circ} 56^{\prime} \mathrm{N}$ ). It is therefore assumed that, during this interval, there was no marked change in the ultraviolet emission from the sun and that the observed small differences in ultraviolet intensities, discussed in the following paragraph, are owing to differences in atmospheric conditions at the two stations.

A comparison of the ultraviolet intensities for the same air masses $(m)$ at the Washington and the San Juan stations, shows a difference of 10 percent for $m=2$ and 7 percent for $m=1.5$ in favor of the San Juan station. While this small difference in ultraviolet intensity may be attributed to greater local air pollution by dust and smoke at the inland, Washington station, it is more likely to be owing to the greater amount of atmospheric ozone in the higher latitude, as indicated by the greater difference in intensities at air mass, $m=2$, as compared with $m=1.5$.

Interesting measurements were made on January 24, 1935, which was an exceedingly clear day, with a noon-hour temperature of $12^{\circ}$ $\mathrm{F}$., with snow on the ground and with the trees covered with ice. The total solar intensity was $?=1.47 \mathrm{~g}$ cal per $\mathrm{cm}^{2}$ per minute, the highest seasonal record, in years, in Washington. This high total radiation intensity is accounted for on the basis of a low pressure of aqueous vapor, which is very opaque to infrared rays and, hence, is most effective in summer.

The ultraviolet filter transmissions were unseasonably high, corresponding with a high ozone content (equivalent to a layer of the pure gas, $3 \mathrm{~mm}$ in thickness, at normal temperature and pressure (ntp), see fig. 7) such as might be expected if the prevailing low temperature was instrumental in conserving the ozone. Incidentally it 
is to be noted that since atmospheric ozone reduces the total incident solar radiation by only 1 part in 1,000 , a slight increase in ozone, which greatly affects the ultraviolet at 2900 to $3200 \mathrm{~A}$, has but little effect upon the total solar intensity. Hence there is nothing inconsistent in our observation of a relatively low ultraviolet intensity and the Weather Bureau measurement of a high total intensity on January 24, 1935.

The filter transmission measurements made at San Juan, especially during the first part of February, are definitely lower for the same air mass than in Washington, indicating a greater intensity of short wave length ultraviolet in the tropics. This is to be ascribed to a smaller amount of ozone in the tropical atmosphere (figs. 7 and 8).

In this connection it is of interest to record that on the basis of published data (14) giving the local seasonal amount of ozone present at different latitudes, the atmospheric ozone at Washington is a minimum (thickness of layer equivalent to about $2.2 \mathrm{~mm}$, ntp) in September-October and a maximum (about $2.8 \mathrm{~mm}$, ntp) in FebruaryMarch-April. On this basis the ozone at San Juan would amount to about $2.0 \mathrm{~mm}$ (ntp) in January-February, rising to 2.1 to $2.2 \mathrm{~mm}$ (ntp) by the middle of March.

Plotting as ordinates the transmission data, for a given filter (e. g., $\mathrm{Ba}-1)$, observed at Washington, at a particular air mass $(m=$ 1.5 ; also $m=2.0$ and $m=2.5$ ), at the two above-mentioned seasons; and plotting as abscissas the corresponding amounts of ozone at these two seasons, and extrapolating these transmission curves (for different air-masses, $m=1.5,2.0,2.5)$ to an ozone content of 2.0 , gives the percentage transmission to be expected at San Juan-the circles, ,oo, in figure 7. It is interesting to record that the percentage transmission of the filters, observed during the first part of February, are in very close agreement with the predicted values, for an ozone content of 1.9 to $2.0 \mathrm{~mm}$, (equivalent thickness (ntp))-figure 7. Moreover, as the season advanced the average values of the transmissions of the filters became unmistakably higher (see fig. 8) particularly by the middle of March, in agreement with the expected seasonal increase in the amount of ozone (2.1 to $2.2 \mathrm{~mm}$ ) in the tropics, and corresponding with the Washington transmissions, observed in September-October, when the estimated amount of ozone is equivalent to a thickness of about $2.2 \mathrm{~mm}$ (ntp).

From the fact that there is less ozone in the atmosphere in the tropics than at higher latitudes, and from the above-mentioned differences in the direct measurements of solar intensities at the two stations, it appears that, for the same solar altitude (air mass traversed) the intensity of short wave length ultraviolet solar radiation is slightly greater at a sea-level station in the tropics than at a similar station in higher altitudes.

However the slight difference in spectral quality and total intensity of ultraviolet solar radiation, for the same solar altitude in the tropics and at higher latitudes, is not the only factor to be considered in connection with differences in physiological effects (e. g., practically no rickets in the tropics) that are observed in different latitudes.

For, in addition to differences in air movement, temperature, humidity, amount of clothing worn, and hours of sunshine per year, it is to be noted that in midlatitude (Washington) during only 2 months of the year (May 15 to July 15) does the sun shine through an 
atmosphere of air-mass, $m=1.04$ to $m=1.05$ (never less than $m=1.04$ ) at the noon hour, whereas in the tropics (San Juan) during almost 6 months, at the noon-hour the sun shines through an air mass of $m=1.05$ or less. Twice during this interval (about May 12 and about August 1, at San Juan) the sun is in the zenith, $m=1.0$; and during an interval of about 4 months (April 20 to August 20) at the noon hour the sun shines through an air mass of $m=1.00$ to 1.01. Consequently during this period not only is the spectrum richer in the shortest wave lengths, but also the total intensity of the short wave length ultraviolet solar radiation at San Juan, at the noon hour, is about 15 percent higher than for air-mass $m=1.05$, or (owing to the latitude effect), about 20 percent higher than is ever attained in Washington-a phenomenon that can occur at a sea-level station only in the tropics. In higher latitudes it is necessary to ascend to high elevations to attain

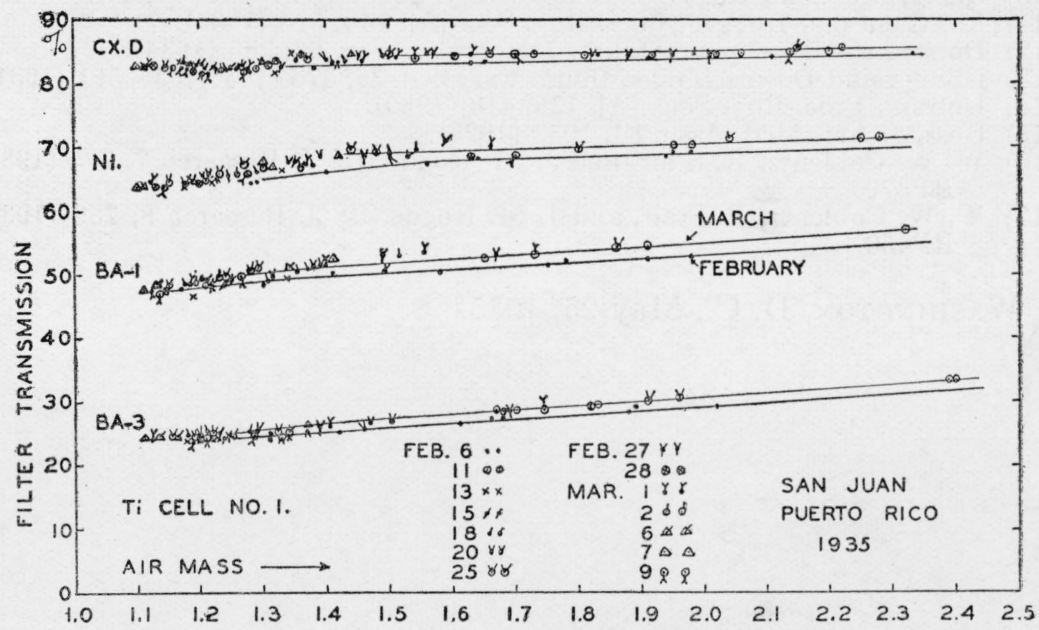

FIG. 8.-Diurnal variation in transmission of ultraviolet solar radiation through standard filters (see fig. 3 ), observed at San Juan, P. R.

this increase in intensity. At the Flagstaff station, for $m=1.05$ the ultraviolet intensities were 40 to 50 percent higher than at Washington.

It is equally interesting to compare ultraviolet intensities during the winter months. At the winter solstice (Dec. 21) the maximum air mass traversed by the solar rays, at the noon hour, is $m=1.34$ at San Juan and $m=2.1$ at Washington. Between October 1 and March 15, the air mass traversed by the solar rays at the noon-hour, at Washington, is always greater than $m=1.34$ and increases to $m=2.1$ in December, at which time the intensity is about one-sixth the value. that obtains when $m=1.34$ and only about one-tenth the summer value when $m=1.04$.

From this it can be seen that in the 5 winter months, when the short wave-length ultraviolet in sunlight is most needed in preventing rickets, the shortest and most effective wave lengths are almost completely absorbed at a midlatitude station (Washington) and the intensity of the less effective wave lengths is less than one-sixth the value that obtains in the tropics. In addition to this fact the actual number of hours of sunshine must be considered (15)-ranging (in the 
5 winter months) from a total of about 200 hours in London and Stockholm, 300 to 350 hours in Berlin and Paris, 800 hours in New York and Colon, and 1,100 hours in San Juan.

\section{REFERENCES}

(1) W. W. Coblentz and R. Stair, BS J. Research 12, 231 (1934) RP647.

(2) Pettit, Astrophys. J. 75, 195 (1932).

(3) Fabry and Buisson, Astrophys. J. 54, 297 (1921).

(4) Kidson, Proc. Roy. Soc. [A] 129, 421 (1930).

(5) W. W. Coblentz, R. Stair, and J. M. Hogue, BS J. Research 10, 79 (1933) RP517.

(6) W. W. Coblentz, R. Stair, and J. M. Hogue, BS J. Research 6, 951 (1931) RP318.

(7) Goetz and Ladenburg, Naturwissenschaften, 19, 373 (1931).

(8) Dobson and Harrison, Proc. Roy. Soc. [A], 110, 660 (1926).

(9) Regener, Phys. Z. 35, 788 (1934).

(10) Goetz, Meetham and Dobson Proc. Roy. Soc. [A], 145, 416 (1935).

(11) Meetham and Dobson, Proc. Roy. Soc. [A], 148, 598 (1935).

(12) Dobson and Meetham, J. Roy. Meteorolog. Soc. 60, 265 (1934).

(13) Greider and Downs, Trans. Illum. Eng. Soc. 25, 378 (1930); 26, 561 (1931).

(14) Dobson, Proc. Roy. Soc., [A], 129, 418 (1930).

(15) Hess, J. Am. Med. Assn. 84, 1033 (1925).

(16) W. W. Coblentz, R. Stair, and J. M. Hogue, BS J. Research 7, 723 (1931) RP370.

(17) W. W. Coblentz, R. Stair, and J. M. Hogue, BS J. Research 8, 759 (1932) RP450.

Washington, D. C., May 25, 1935. 